ARTICLE

https://doi.org/10.1038/s41467-019-08405-9

\title{
Corticosteroids inhibit Mycobacterium tuberculosis- induced necrotic host cell death by abrogating mitochondrial membrane permeability transition
}

Jessica Gräb ${ }^{1,2}$, Isabelle Suárez ${ }^{1,3}$, Edeltraud van Gumpel ${ }^{1,2}$, Sandra Winter ${ }^{1,2}$, Fynn Schreiber ${ }^{1,2}$, Anna Esser ${ }^{1,2}$, Christoph Hölscher ${ }^{3,4}$, Melanie Fritsch 2,5,6, Marc Herb ${ }^{5,6}$, Michael Schramm ${ }^{5,6}$, Laurens Wachsmuth7, Christian Pallasch 1,5, Manolis Pasparakis (10) 2,5,7, Hamid Kashkar 2,5,6 \& Jan Rybniker 1,2,3

Corticosteroids are host-directed drugs with proven beneficial effect on survival of tuberculosis (TB) patients, but their precise mechanisms of action in this disease remain largely unknown. Here we show that corticosteroids such as dexamethasone inhibit necrotic cell death of cells infected with Mycobacterium tuberculosis (Mtb) by facilitating mitogen-activated protein kinase phosphatase 1 (MKP-1)-dependent dephosphorylation of p38 MAPK. Characterization of infected mixed lineage kinase domain-like (MLKL) and tumor necrosis factor receptor 1 (TNFR1) knockout cells show that the underlying mechanism is independent from TNF $\alpha$-signaling and necroptosis. Our results link corticosteroid function and p38 MAPK inhibition to abrogation of necrotic cell death mediated by mitochondrial membrane permeability transition, and open new avenues for research on novel host-directed therapies (HDT).

\footnotetext{
${ }^{1}$ Department I of Internal Medicine, University of Cologne, 50937 Cologne, Germany. ${ }^{2}$ Center for Molecular Medicine Cologne (CMMC), University of Cologne, 50931 Cologne, Germany. ${ }^{3}$ German Center for Infection Research (DZIF), Partner Site Bonn-Cologne, Cologne, Germany. ${ }^{4}$ German Center for Infection Research (DZIF), Partner Site Hamburg - Lübeck - Borstel - Riems 23845, Germany. ${ }^{5}$ Excellence Cluster on Cellular Stress Responses in AgingAssociated Diseases (CECAD), University of Cologne, 50931 Cologne, Germany. ${ }^{6}$ Institute for Medical Microbiology, Immunology and Hygiene (IMMIH), University of Cologne, 50935 Cologne, Germany. ${ }^{7}$ Institute for Genetics, University of Cologne, 50674 Cologne, Germany. Correspondence and requests for materials should be addressed to J.R. (email: jan.rybniker@uk-koeln.de)
} 
M ycobacterium tuberculosis $(M t b)$, the causative agent of tuberculosis (TB), is the major killer among infectious agents which led to 1.7 million deaths in 2016 (ref. ${ }^{1}$ ). Clinical management of $\mathrm{TB}$ consists of combinations of antibiotics for several months, a concept which becomes increasingly complicated in times of rising numbers of multi-drug resistant $M t b$-isolates ${ }^{1}$. Adjunctive host-directed therapy (HDT) might improve and accelerate treatment by modifying host pathways targeted by the intracellular pathogen $M t b^{2-6}$. One possible approach is to inhibit cell death induced by phagocytosed $M t b$ which can prevent mycobacterial spread, tissue damage, and hyperinflammation ${ }^{3}$. Successful implementation of such drugs requires fundamental understanding of cell-death mechanisms exploited by $M t b$. However, the molecular pathways involved in the manipulation of host cell death, and the consequence of this on the outcome of the infection, remain highly controversial ${ }^{7-9}$. Since lytic cell death seems to benefit the mycobacteria by enabling dissemination inside the human body and spread to other individuals, current research efforts focus on elucidating the exact mechanisms of $M t b$-induced necrosis, which occurs once intracellular bacteria enter their replicative mode. Initially, in vitro studies as well as data derived from zebrafish infection models suggested that tumor necrosis factor alpha (TNFa) and mixed lineage kinase domain-like- (MLKL) driven necroptosis is the main cell-death pathway exploited by $M t b^{10,11}$. These findings were linked to clinical data showing hyperinflammation and increased mortality in Vietnamese patients with TB meningitis (TBM) carrying a specific leukotriene A4 hydrolase (LTA4H) promoter genotype leading to elevated TNFa levels ${ }^{11,12}$. However, this LTA4H genotype was not associated with increased severity and frequency of pulmonary TB in non-Asian patients ${ }^{13}$. A recent clinical study performed in Indonesia found no correlation between LTA4H promoter polymorphism and TBM outcome $^{14}$. Another study showed that necroptotic signaling is primed in $M t b$-infected macrophages, but its pathophysiological consequence in disease is restricted ${ }^{15}$. Therefore, while it is clear that host cell death significantly affects the outcome of $\mathrm{TB}$, it is critically important to understand the precise role of TNFa signaling and necroptosis in the pathogenesis of $M t b$ to effectively interfere with these pathways by HDT.

Currently, corticosteroids, such as dexamethasone, represent the only clinically approved adjunctive chemotherapeutics for $\mathrm{TB}^{3,16}$. However, their exact mechanism of action is ill-defined despite proven beneficial effect on survival of TB patients ${ }^{17}$. It is assumed that corticosteroids function via systemic suppression of $\mathrm{TNF} \alpha$ in hyper-inflammatory states of the disease ${ }^{12}$. A direct cytoprotective effect of corticosteroids, which are extensively used in multiple other inflammatory diseases, has not been described so far.

Exploiting a high-throughput chemical genetics approach selecting for small molecules that abrogate $M t b$-induced death of human phagocytes, we identified several clinically applied corticosteroids that protected $M t b$-infected cells as efficiently as antituberculous antibiotics.

This key finding here provides an excellent opportunity to gain fundamental insight into mechanisms of $M t b$-induced host cell death and its impact on a clinically approved therapeutic intervention. Using corticosteroids as a starting point, we systematically investigated cellular machineries executing apoptosis, necroptosis, and alternative forms of regulated necrosis. Our mechanistic studies identified the mitochondrial permeability transition pore (mPTP) to be involved in necrotic cell death upon $M t b$ infection. The data obtained suggest that $M t b$ controls necrosis by manipulating mitochondrial membrane integrity and successful therapeutic interventions ultimately target mitochondria and interfere with TB pathogenesis.

\section{Results}

Corticosteroids potently inhibit $M$ tb-induced cell death. Coincubation and infection of phagocytes with $M t b$ inevitably leads to host cell death which is primarily mediated by the mycobacterial ESX-1 type VII secretion system, an essential virulence factor $^{18}$. Massively attenuated mycobacteria such as the Bacille Calmette-Guérin vaccine strain fail to kill host cells which makes $M t b$-induced cell death or cell survival an attractive readout for host-directed drug screens ${ }^{18}$. When testing pharmacologically active and approved drugs in a high-throughput chemical genetic screen selecting for compounds that abrogate $M t b$-dependent cell death ${ }^{19,20}$, we detected a series of corticosteroids as potent hit compounds that protected $M t b$-infected MRC-5 human lung fibroblasts as efficiently as known anti-mycobacterial drugs (Fig. 1a; Supplementary Fig. 1; Supplementary Table 1). The corticosteroid dexamethasone was highly cytoprotective even at low nanomolar concentrations $\left(\mathrm{IC}_{50}: 15 \mathrm{nM}\right)$ (Fig. 1b). Cell death was also abrogated in $M t b$-infected $\mathrm{J} 774$ mouse macrophages $(\mathrm{M} \varphi)($ Fig. 1c, d) and in human $\mathrm{M} \varphi$ isolated from healthy donors and treatment naïve TB patients (Fig. 1c, e, f). Since corticosteroids are extensively used as adjunctive therapy in TB affecting the central nervous system we also tested for survival of pretreated and infected microglial cells. Chemical treatment with dexamethasone led to strong survival of BV-2 microglial cells comparable to other infected phagocytes (Fig. 1c, g).

Dexamethasone abrogates p38 MAPK phosphorylation. The corticosteroid-inducible protein mitogen-activated protein kinase (MAPK) phosphatase 1 (MKP-1; DUSP1) has emerged as a key molecule responsible for steroid-dependent effects on eukaryotic cells $^{21}$. We speculated that inhibition of MKP-1 negatively affects the cytoprotective effects that were observed in dexamethasonetreated cells. Co-treatment of $M t b$-infected human lung fibroblasts with dexamethasone and the specific MKP-1 inhibitor (E/Z)-BCI fully abrogated the dexamethasone protective effect (Fig. 2a). The substance alone showed no growth inhibitory effect on host cells (Fig. 2a). Similar observations were made when cells were co-treated with dexamethasone and the glucocorticoid receptor (GR) inhibitor Ru-486 or by single-agent treatment with the selective non-steroidal GR agonist BI653048 indicating that dexamethasone protects the cells via GR activation and MKP-1 upregulation (Fig. 2a, b; Supplementary Fig. 2). Strong MKP-1 upregulation of dexamethasone-treated $M t b$-infected cells was confirmed by quantitative reverse transcriptase polymerase chain reaction (qRT-PCR) (Fig. 2c). MKP-1 specifically inactivates p38 MAPK, c-Jun N-terminal kinases (JNK), and extracellular signalregulated kinases $(\mathrm{ERK})^{22}$. We tested for phosphorylation of these kinases upon infection with $M t b$ and found p38 MAPK phosphorylation at several time points after infection of human lung fibroblasts and J774 M $\varphi$ (Fig. 2d-f). Dexamethasone treatment of infected cells inhibited p38 MAPK phosphorylation in both cell types (Fig. 2d-f; Supplementary Fig. 3). JNK or ERK phosphorylation was not observed at 5 and $24 \mathrm{~h}$ post infection (Supplementary Fig. 4a and b).

Phosphorylation of p38 MAPK initiates cell death. Having identified p38 MAPK as a putative target of corticosteroids, we tested a series of p38 MAPK inhibitors and examined the survival of $M t b$-infected cells. The data obtained showed that pamapimod, BMS-582949 and SB203580 did not interfere with $M t b$-induced cell death at $10 \mu \mathrm{M}$ (Supplementary Fig. 5a). In contrast, the highly potent and selective p38 MAPK inhibitor doramapimod was as protective as dexamethasone in several infected cell types including human $\mathrm{M} \varphi$ isolated from healthy donors and treatment naïve TB patients (Fig. 2g, h; Supplementary Fig. 5b-e). To better 

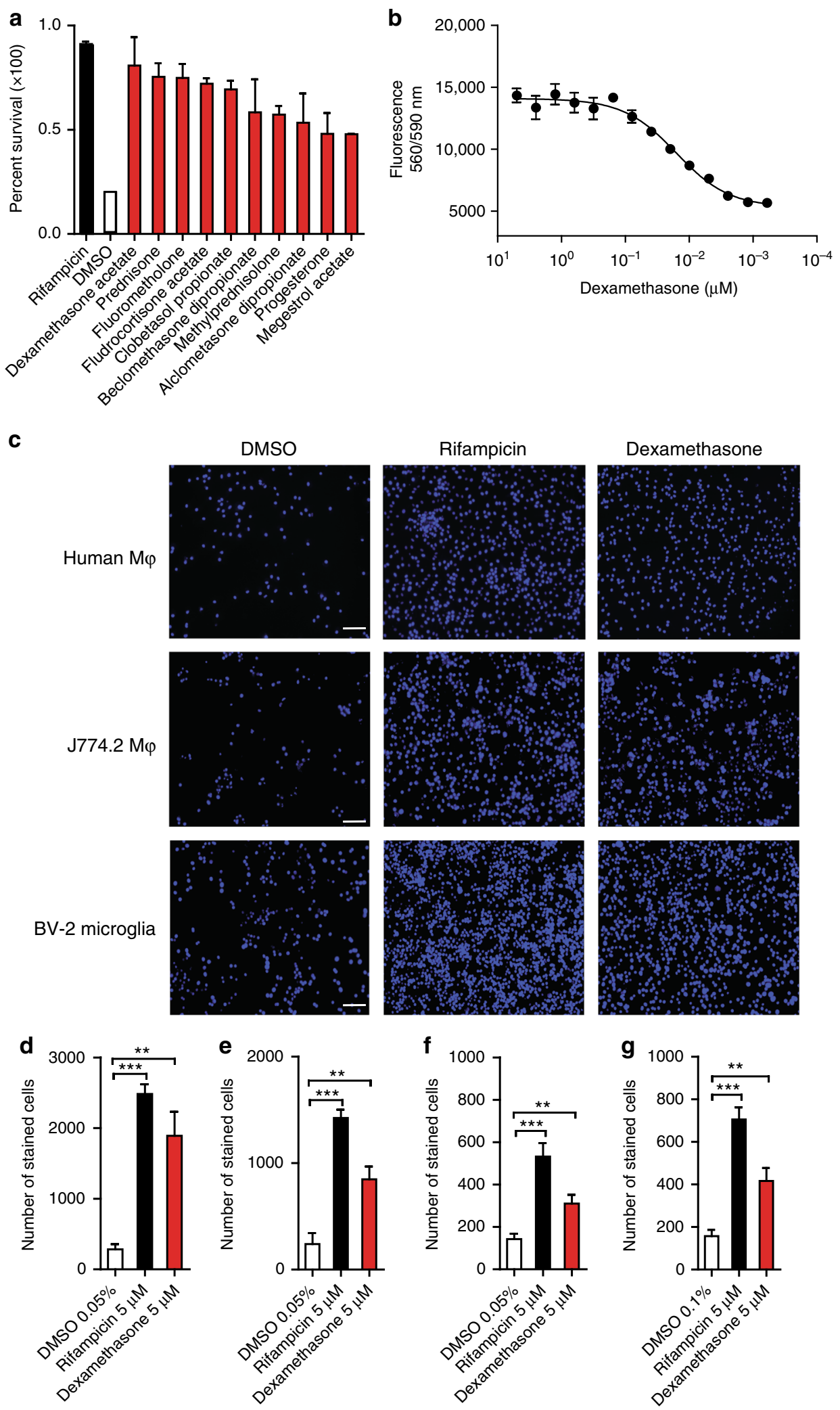

understand these conflicting results we quantified p38 MAPK phosphorylation in BMS-582949 and doramapimod-treated MRC-5 lung fibroblasts after infection with $M t b$. Interestingly, only doramapimod was capable of suppressing p38 MAPK phosphorylation in a sustainable manner whereas BMS-582949 failed to inhibit phosphorylation at 24 and $48 \mathrm{~h}$ post infection

(Fig. 2i). This indicates that $M t b$ infection represents a potent inflammatory stimulus that can overcome chemical p38 MAPK inhibition in some of these substances. The underlying molecular mechanism however remains elusive.

To definitely clarify the role of p38 MAPK in $M t b$-induced cell death, the expression of p38 MAPK was down regulated (p38KD) 
Fig. 1 Corticosteroids abrogate cytotoxicity in Mycobacterium tuberculosis (Mtb)-infected cells. a Protective effect of dexamethasone and other corticosteroids (10 $\mu \mathrm{M})$ in Mtb-infected MRC-5 lung fibroblasts (multiplicity of infection (MOI) 10). Host cell viability was quantified using PrestoBlue. Data derived from high-throughput screen with measurements of duplicate assay plates. b Dose-response curve of dexamethasone in Mtb-infected MRC-5 lung fibroblasts analyzed using the fibroblast survival assay (FSA). c Representative fluorescent microscopy images of Mtb-infected primary human

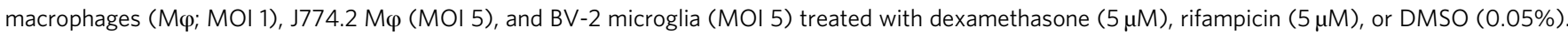
Nuclei were stained with 4',6-diamidino-2-phenylindole (DAPl; scale bar: $100 \mu \mathrm{m}$ ). Images are representative of two to three individual experiments with multiple replicates. $\mathbf{d}-\mathbf{g}$ Dexamethasone $(5 \mu \mathrm{M})$-treated $\mathrm{J} 774.2$ mouse $\mathrm{M} \varphi(\mathbf{d})$, primary human $\mathrm{M} \varphi$ from healthy donors (e) and TB patients (f), and BV-2 mouse microglia $(\mathbf{g})$ were infected with the wild-type Mtb strain Erdman at varying MOI and surviving cells were stained with DAPI to determine the number of living cells $48 \mathrm{~h}$ post infection. Data from one experiment with duplicates are shown in $\mathbf{b}$; data were pooled from two (d, $\mathbf{f}, \mathbf{g}$ ) or three (e) independent experiments with multiple replicates. Results are expressed as the mean \pm SEM. Statistical analysis were performed by unpaired $t$-test $\left.{ }^{\star \star \star} p \leq 0.01 ;{ }^{\star \star \star} p \leq 0.001\right)$

in J774 M $\varphi$ using selective siRNAs (Supplementary Fig. 6). Down-regulation of p38 expression significantly increased the survival of infected $\mathrm{J} 774 \mathrm{M} \varphi$, clearly indicating that p38 MAPK is involved in $M t b$-induced cell death (Fig. 2j).

Apoptosis is not the main mechanism for p38 MAPK mediated cell death. To better understand the events following p38 MAPK phosphorylation as a consequence of $M t b$ infection, we carefully dissected several cell death pathways. MAP-kinases have been frequently associated with apoptotic processes such as the activation of pro-apoptotic Bcl-2 family proteins leading to caspase activation ${ }^{7}$. We first determined activation of executioner caspases in our infection models using immunoblots targeting cleaved caspase 3. $M t b$ infection led to some proteolytic activation of caspase 3 in $\mathrm{J774} \mathrm{M} \varphi$ which was partially inhibited by dexamethasone and doramapimod (Fig. 3a). In a more sensitive luminescence-based caspase activity assay for both caspase 3 and caspase 7, a stronger activation signal was detected upon infection of human lung fibroblasts (Fig. 3b). p38 MAPK inhibition via dexamethasone or doramapimod led to a slight decrease in luminescent signal (Fig. 3b). We then treated cells with the pancaspase inhibitor Z-VAD-FMK ( $N$-benzlyoxycarbonyl-valyl-alanyl-aspartyl-fluoromethylketone). This substance led to full inhibition of caspase 3- and caspase 7 activation in infected cells (Fig. 3b). Assuming that p38 MAPK signaling leads to apoptosis execution via caspase $3 / 7$, Z-VAD-FMK should be as cytoprotective as dexamethasone or doramapimod. However, caspase inhibition by Z-VAD-FMK failed to protect human lung fibroblasts and human primary $\mathrm{M} \varphi$ from $M t b$-induced cell death (Fig. 3c, d) excluding apoptosis as a responsible killing mechanism upon $M t b$ infection and indicating that caspase 3 activation is an incidental event of cell stress.

p38 MAPK inhibition blocks secretion of necrosis markers. There is growing evidence that necrotic cell death plays a key role in pathogen-driven inflammation, cell to cell spread, and dissemination to new hosts ${ }^{23}$. We found large amounts of lactate dehydrogenase (LDH), a necrosis indicator, in the supernatant of $M t b$-infected lung fibroblasts as well as in human $\mathrm{M} \varphi$ isolated from healthy donors and treatment-naïve TB patient (Fig. 4a, b). In addition, the necrosis marker high-mobility group protein $\mathrm{B} 1$ (HMGB1) was released from $M t b$-infected fibroblasts (Fig. 4c). $\mathrm{LDH}$ and HMGB1 release was blocked in dexamethasone- or doramapimod-treated cells (lung fibroblasts, $\mathrm{M} \varphi$ isolated from TB patients) (Fig. $4 \mathrm{a}-\mathrm{c}$ ). Collectively, these data link p38 MAPK activity to necrosis rather than apoptosis.

TNFa and necroptosis are dispensable for corticosteroid function. The prototype of regulated necrotic cell death is TNFadependent necroptosis involving a microfilament like complex orchestrated by phosphorylated receptor-interacting protein kinase 1 (RIPK1), RIPK3, and MLKL ${ }^{23}$. However, there are conflicting data regarding the role of this cell death mechanism in the pathogenesis of $M t b^{10,15,24,25}$. Since corticosteroids have been shown to interfere with TNFa signaling, we investigated whether specific inhibition of the TNF-receptor (TNFR), TNFa itself, or RIPK1 impacts on susceptibility of cells upon $M t b$ infection. Interestingly, chemical TNFa inhibition using thalidomide, etanercept, or adalimumab as well as RIPK1 inhibition with necrostatins (Nec-1 and Nec-5) failed to protect $M t b$-infected host cells (Fig. 4d; Supplementary Fig. 7). Infected bone-marrowderived monocytes (BMDM) from $M l k l^{-1-}$ mice $^{26}$ were as equally susceptible to $M t b$-dependent cell death as wild-type cells (Fig. 4e). Similar results were obtained in BMDM derived from Tnfr $1^{-1-}$ mice $^{27}$ (Fig. 4f). Of note, chemical inhibition of p38 MAPK retained its cytoprotective activity in these knockout cells (Supplementary Fig. 8). These findings separate corticosteroid and 338 MAPK function from TNFa and necroptosis. Recent data indicate that certain triggers of necroptosis require caspase inhibition for execution of programmed cell death ${ }^{28-30}$. A pronecroptotic milieu can be generated by chemical caspase inhibition, which renders cells susceptible to necrostatin-1 blockade resulting in cell survival. To rule out that this effect occurs in $M t b$-infected cells, we treated human lung fibroblasts with both Z-VAD-FMK and necrostatin-1. This dual treatment had no cytoprotective effect despite caspase activation after $M t b$ infection (Figs. $4 \mathrm{~g}$ and $3 \mathrm{a}, \mathrm{b}$ ).

Host cell protection involves mitochondrial membrane permeability transition. Having established that necroptosis is an unlikely mechanism of p38 MAPK-driven cell death, we investigated alternative causes of regulated necrosis. In-depth evaluation of data derived from our high-throughput chemical genetic screen selecting for compounds that abrogate $M t b$-dependent cell death identified Cyclosporin A (CsA) as a highly cytoprotective hit compound (Supplementary Table 1). CsA inhibits opening of the $\mathrm{mPTP}$ by interacting with the $\mathrm{mPTP}$-regulating protein cyclophilin D (CypD) ${ }^{23}$. CsA, but not FK506, a calcineurin inhibitor with no activity on CypD, was as protective as dexamethasone or doramapimod in our host cell survival assays indicating that mitochondrial membrane permeability transition (MPT) is required for $M t b$-induced cell death (Fig. 5a). Prolonged mPTP opening results in loss of mitochondrial membrane potential $(\Delta \Psi \mathrm{m})$, mitochondrial rupture, and necrotic cell death $^{31}$. This effect was visualized using tetramethylrhodamine ethyl ester (TMRM) staining which detects $\Delta \Psi \mathrm{m}$ changes. The cell permeable TMRM dye accumulated in healthy, non-infected $\mathrm{M} \varphi$ but not in infected $\mathrm{M} \varphi$ giving further proof that $M t b$ kills host cells by mPTP opening which results in disruption of the mitochondrial membrane potential (Fig. 5b). Having identified CypD and the mPTP as a potential target of p38 MAPKdependent host cell death, we speculated that chemical p38 MAPK inhibition might reduce mitochondrial CypD in infected 
a

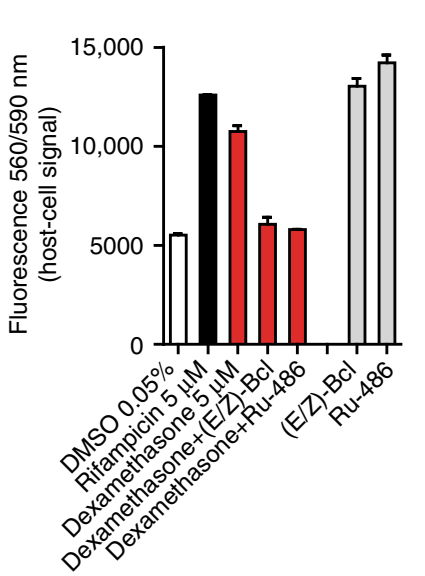

d

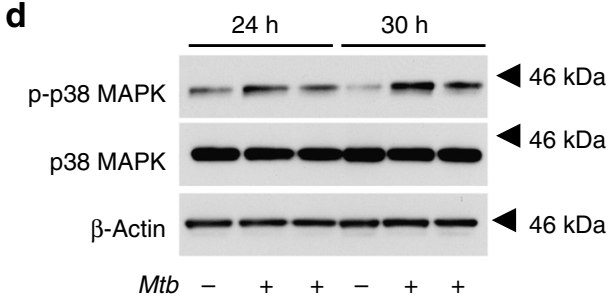

Dexamethasone - $-+-{ }_{-}+$ b
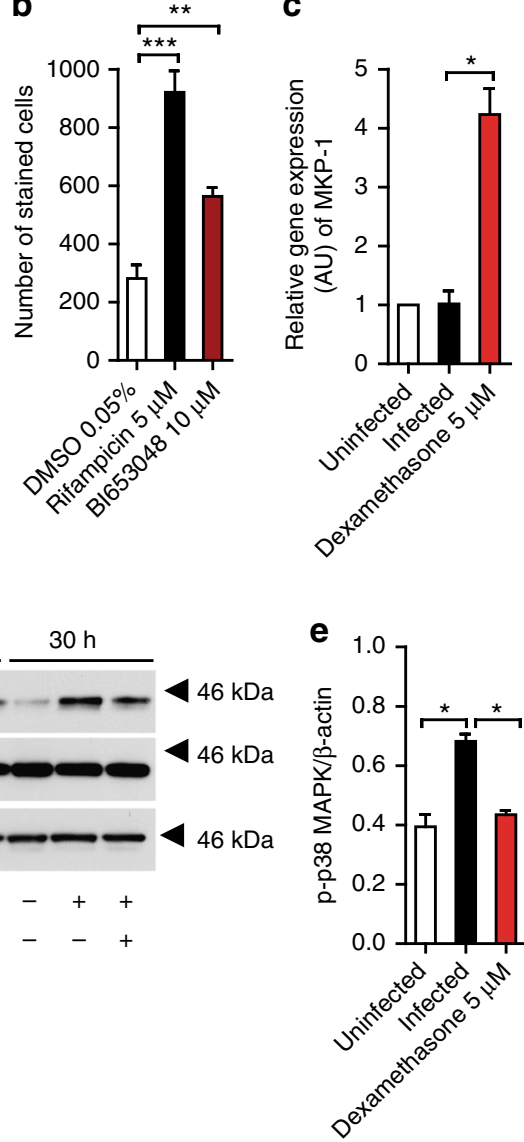

f

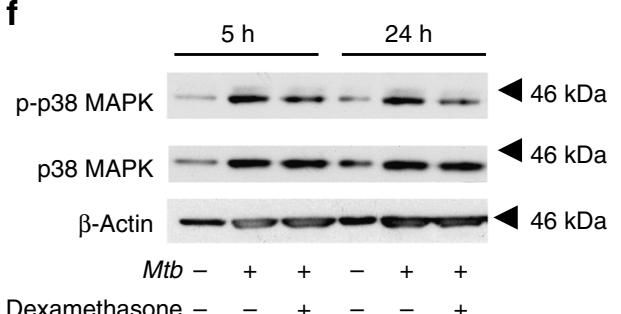

Dexamethasone - $-+\ldots+$

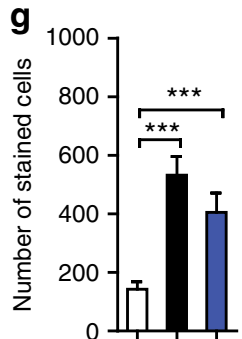

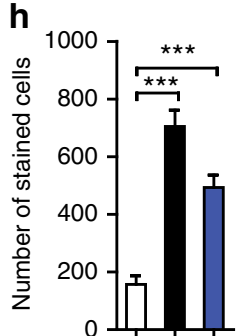
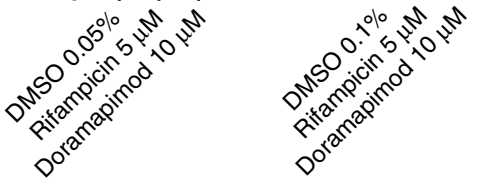

i
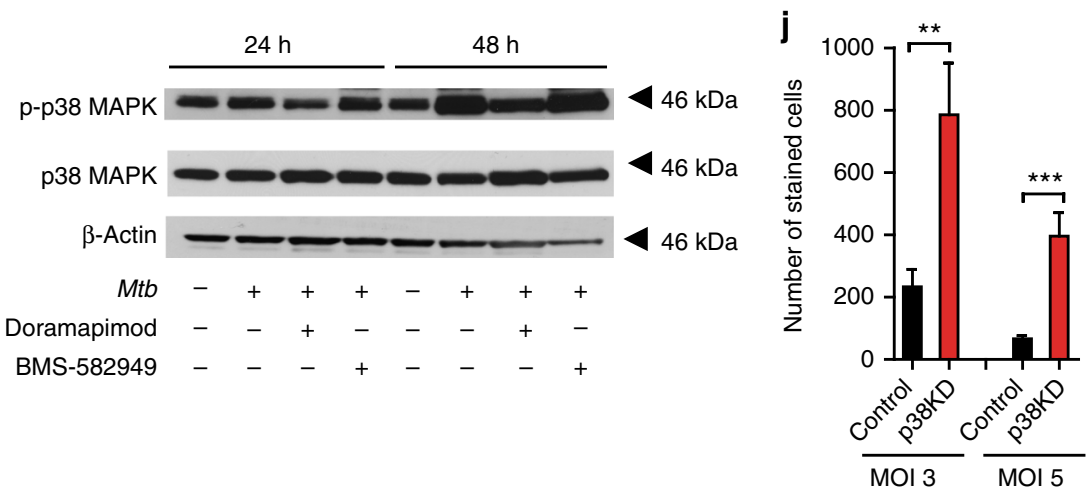
Fig. 2 The protective effect of dexamethasone is mediated by MKP-1 and p38 MAPK inhibition. a Inhibition of MKP-1 by (E/Z)-Bcl hydrochloride or inhibition of the glucocorticoid receptor (GR) by Ru-486 in Mtb-infected MRC-5 lung fibroblasts (MOI 10) abrogates the protective effect of dexamethasone $(5 \mu \mathrm{M})$ on host cell survival. Viable fibroblasts were quantified $72 \mathrm{~h}$ post infection using PrestoBlue. b Protective effect of the GR agonist BI653048 $(10 \mu \mathrm{M})$ in $\mathrm{Mtb}$-infected human $\mathrm{M \varphi}(\mathrm{MOI} 1)$. Cells were stained with DAPI and the number of living cells was determined $48 \mathrm{~h}$ post infection. $\mathbf{c}$ Dexamethasone $(5 \mu \mathrm{M})$ treatment increases MKP-1 expression in infected MRC-5 lung fibroblasts $5 \mathrm{~h}$ after infection. Relative expression of MKP-1 was measured by quantitative real-time PCR (qRT-PCR). d-f Mtb induces p38 MAPK activation in $5774.2 \mathrm{M \varphi}$ (d, e) and MRC-5 lung fibroblasts (f). Whole-cell lysates were obtained from infected cells treated with or without dexamethasone $(5 \mu \mathrm{M})$ and equal amounts of protein were subjected to western blot analysis to determine the levels of phosphorylated and total p38 MAPK. $\beta$-Actin was used as a loading control. Images are representative of two to three individual experiments. $\mathbf{g}, \mathbf{h}$ Cytoprotective effect of the p38 MAPK inhibitor doramapimod $(10 \mu \mathrm{M}$ ) in Mtb-infected human M $\varphi$ from healthy donors ( $\mathbf{g}$ ) and TB patients (h). Cells were stained with DAPI and the number of living cells was determined $48 \mathrm{~h}$ post infection. i Doramapimod (10 $\mu$ M) inhibits $\mathrm{Mtb}$ induced p38 MAPK phosphorylation. Quantification of phosphorylated p38 MAPK in MRC-5 lung fibroblasts infected with Mtb and treated with the p38 MAPK inhibitors BMS-582949 (10 $\mu$ M) or doramapimod by western blot. $\mathbf{j}$ Knock-down of p38 MAPK in Mtb-infected J774A.1 M $\varphi$ reduces mycobacterial cytotoxicity. Infected cells (MOI 3 and 5) were stained with DAPI and the number of surviving cells was determined after $48 \mathrm{~h}$ of infection. Data from one experiment with multiple replicates $(\mathbf{a}, \mathbf{b})$ or data pooled from four individual experiments (c) are shown; data from two individual experiments are shown in $\mathbf{e}$ and $\mathbf{h}$; data from three independent experiments $(\mathbf{g}, \mathbf{j})$ with multiple replicates are shown as mean \pm SEM. Analysis was done using unpaired $t$ - test $\left({ }^{\star} p \leq 0.05 ;{ }^{* \star} p \leq 0.01 ;{ }^{* \star *} p \leq 0.001\right)$

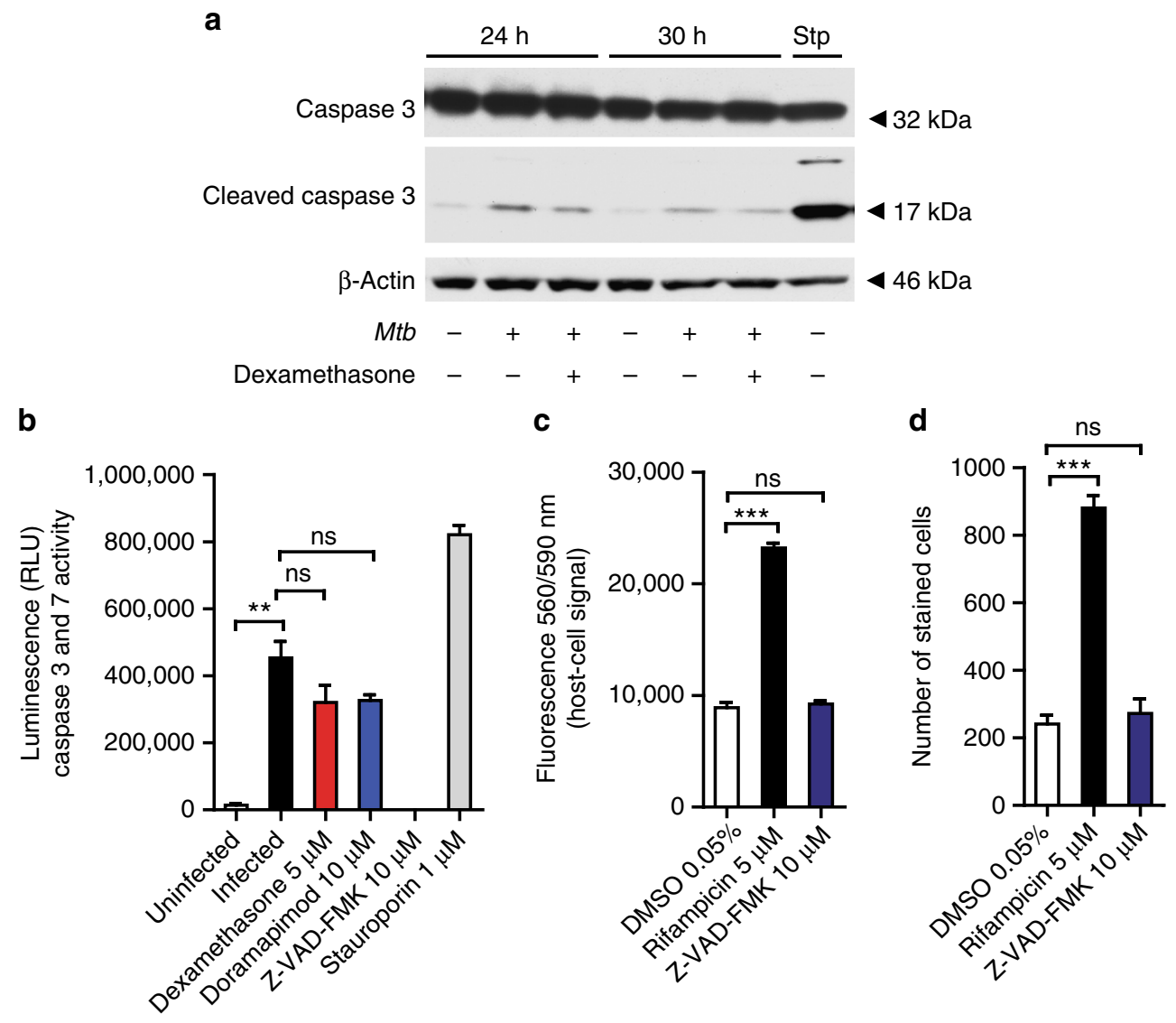

Fig. $3 \mathrm{Mtb}$-induced cell death is independent of caspase activation. a Western blot analysis of proteolytic cleavage of caspase 3 in $\mathrm{Mtb}$-infected J774.2 M $\mathrm{\varphi}$ (MOI 5). Equal amounts of protein from infected J774.2 M $\varphi$ treated with or without dexamethasone (5 $\mu \mathrm{M}$ ) were subjected to immunoblot analysis for cleaved caspase 3 following 24 and $30 \mathrm{~h}$ of infection. $\beta$-Actin was used as a loading control and staurosporine $(1 \mu \mathrm{M})$ as a positive control. Images are representative of two individual experiments. $\mathbf{b}$ MRC-5 lung fibroblasts were infected with $M t b(\mathrm{MOI} 10)$ and treated with dexamethasone $(5 \mu \mathrm{M})$, doramapimod $(10 \mu \mathrm{M})$, or Z-VAD-FMK $(10 \mu \mathrm{M})$. After $48 \mathrm{~h}$ caspase 3 and caspase 7 activity was assessed using a luminescent probe. Uninfected and staurosporine $(1 \mu \mathrm{M})$-treated cells were used as controls. c, $\mathbf{d}$ Effect of caspase 3 and caspase 7 inhibition using the pan-caspase inhibitor Z-VAD-FMK (10

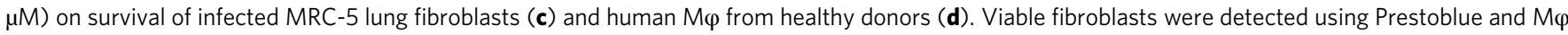
were quantified by DAPI staining. Representative data from two experiments with multiple replicates are shown in b-d. Results are expressed as mean \pm SEM. Analysis was done using unpaired $t$-test (ns not significant; ${ }^{\star \star} p \leq 0.01 ;{ }^{* \star} p \leq 0.001$ )

cells. However, using CsA as a control, we did not observe decreased CypD levels in doramapimod or dexamethasone treated and $M t b$-infected cells (Fig. 5c; Supplementary Fig. 9). Another important regulator of $\mathrm{mPTP}$ opening is the glycolytic enzyme hexokinase II (HKII). While not directly involved in pore formation, translocation and increased binding of HKII to mitochondria protects cells from stress induced by mPTP opening $^{32}$. Relative gene expression analysis showed that HKII is significantly up-regulated $5 \mathrm{~h}$ after $M t b$ infection (Fig. $5 \mathrm{~d}$ ). At this early time-point of infection, mitochondrial HKII levels were higher in infected versus non-infected J774 M $\varphi$ (Fig. 5e; Supplementary Fig. 10). However, at $24 \mathrm{~h}$ post infection HKII levels 
a

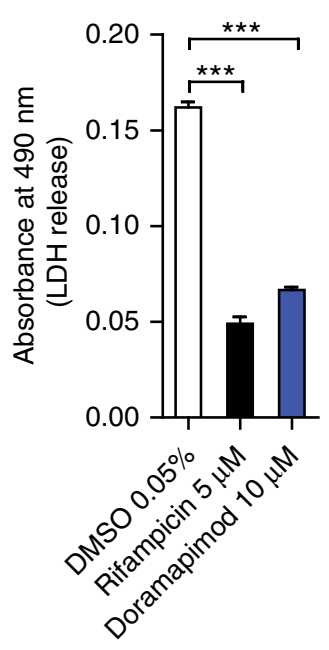

e

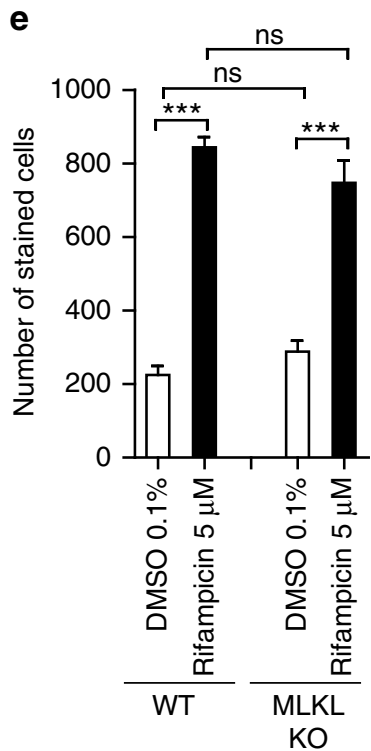

b

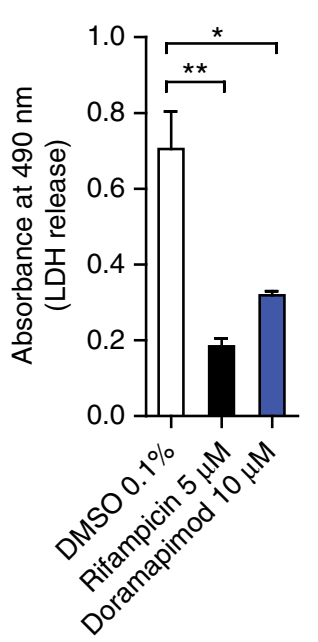

C

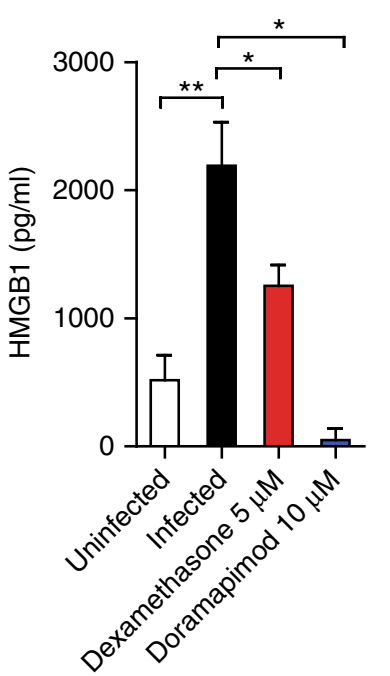

d

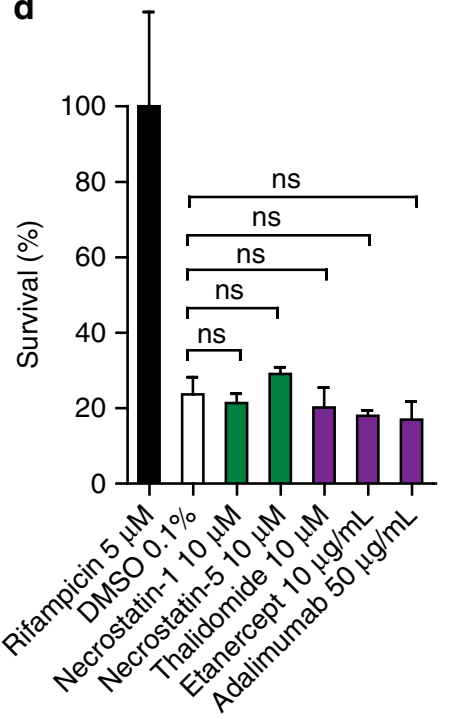

f

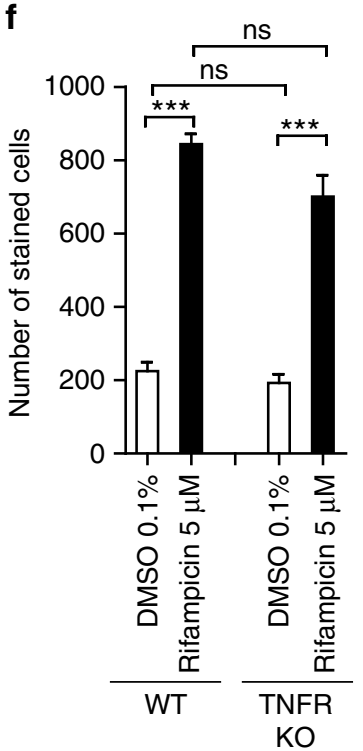

g

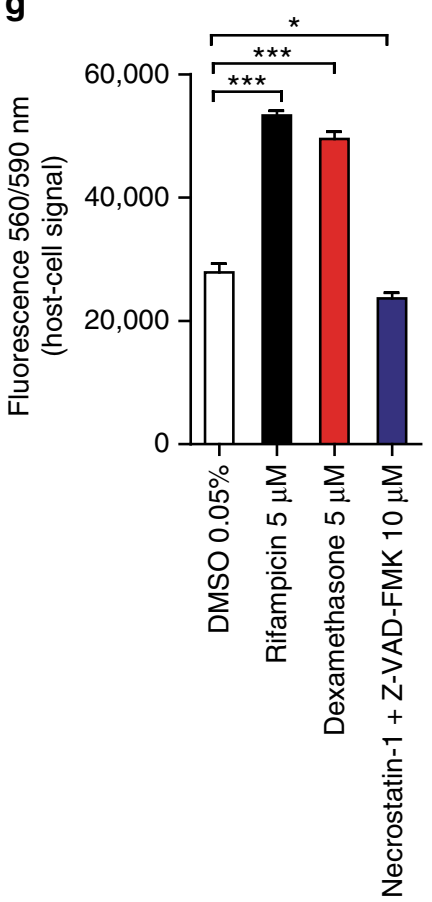

Fig. $4 \mathrm{Mtb}$ induces RIPK1 independent necrotic host cell death. a, b Mtb-infected MRC-5 lung fibroblasts were treated with doramapimod (10 $\mu$ M). After $72 \mathrm{~h}$ lactate dehydrogenase (LDH) was quantified in the culture medium of infected cells (a). Mtb-infected human M $\varphi$ derived from TB patients were treated with doramapimod and LDH release was measured $48 \mathrm{~h}$ post infection in the supernatant (b). Data are representative of two individual experiments. c Dexamethasone $(5 \mu \mathrm{M})$ and doramapimod $(10 \mu \mathrm{M})$ reduce the release of the necrosis marker High-Mobility Group Protein B1 (HMGB1) in Mtb-infected MRC-5 lung fibroblasts. d Protective effect of necrostatin ( Nec; $10 \mu \mathrm{M})$, etanercept ( $10 \mu \mathrm{g} / \mathrm{ml})$, adalimumab (50 $\mu \mathrm{g} / \mathrm{ml})$, and thalidomide $(10 \mu \mathrm{M})$ in $\mathrm{Mtb}$ infected human M $\varphi$ from healthy donors. Cell viability was quantified by DAPI staining $48 \mathrm{~h}$ after infection. e Bone-marrow-derived M $\varphi$ (BMDM) from WT and mixed lineage kinase domain-like pseudokinase $(\mathrm{MLKL})^{-/-}$mice were infected with $\mathrm{Mtb}(\mathrm{MOI} 10)$ and cell survival was assessed $48 \mathrm{~h}$ after infection using DAPI staining. f BMDM from WT and tumor necrosis factor receptor (TNFR) ${ }^{-/-}$mice was infected with Mtb (MOI 10). Cell survival was determined $48 \mathrm{~h}$ after infection using DAPI staining. $\mathbf{g}$ MRC-5 lung fibroblasts were treated with Z-VAD-FMK $(10 \mu \mathrm{M})$ and necrostatin-1 (10 $\mu \mathrm{M})$ simultaneously and viable cells were analyzed $72 \mathrm{~h}$ post infection with TB using PrestoBlue. Representative data from at least two experiments with multiple replicates are shown in $\mathbf{a}, \mathbf{b}, \mathbf{d}$-f. And data pooled from eight independent experiments are shown in $\mathbf{c}$. Representative data from two experiments with multiple replicates are shown in $\mathbf{g}$. Results are expressed as mean \pm SEM. Analysis was done using unpaired $t$-test (ns not significant; ${ }^{*} p \leq 0.05 ;{ }^{\star \star} p \leq 0.01 ;{ }^{\star \star \star} p \leq 0.001$ )

declined indicating that infected cells are not capable of maintaining protective HKII levels on the mitochondrial membrane (Fig. 5e). Intriguingly, dexamethasone and doramapimod treatment led to sustainably increased levels of mitochondrial HKII in infected cells (Fig. 5e). In human lung fibroblasts we observed a strong increase of cytosolic HKII $48 \mathrm{~h}$ after infection indicating significant HKII dissociation from the mitochondrion (Fig. 5f; Supplementary Fig. 11). This effect was fully abrogated in doramapimod-treated cells but not in cells treated with BMS582949, the substance that fails to dephosphorylate p38 MAPK 
a

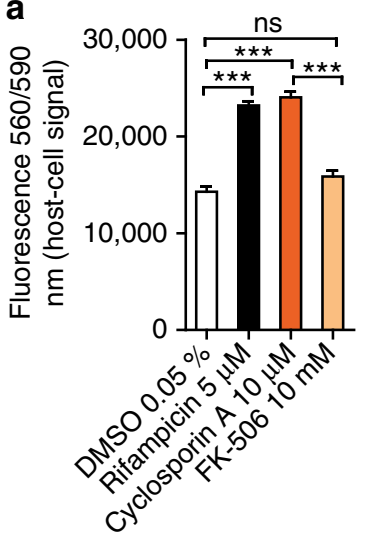

c

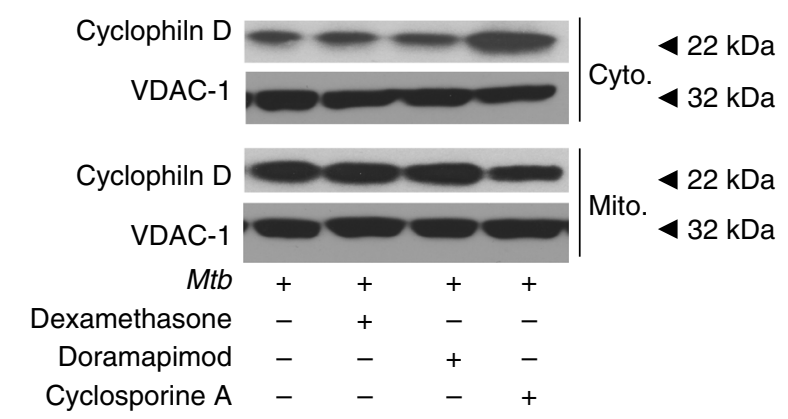

b

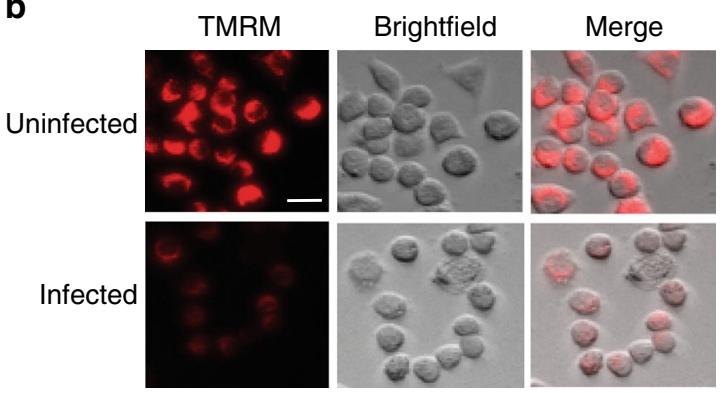

e

$5 \mathrm{~h}$

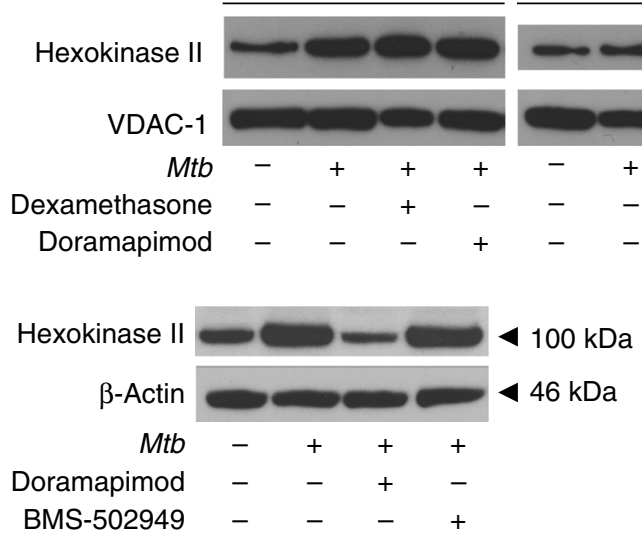

d

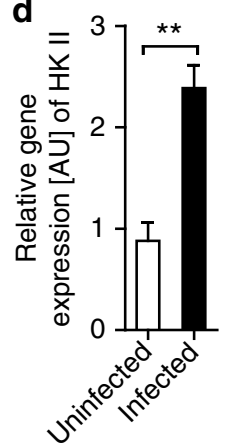

$24 \mathrm{~h}$

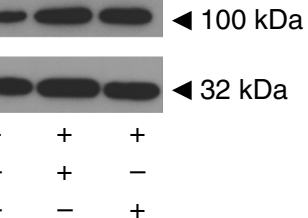

Fig. 5 Mtb triggers p38 MAPK-dependent necrosis by opening of the mitochondrial permeability transition pore (mPTP). a Protective effect of cyclosporine A (CsA; $10 \mu \mathrm{M})$ in Mtb-infected MRC-5 lung fibroblasts (MOI 10). Viability was quantified $72 \mathrm{~h}$ after infection using Prestoblue. $\mathbf{b}$ Fluorescence microscopy of uninfected and infected (MOI 5) J774.2 M $\varphi$ stained with tetramethylrhodamine (TMRM) $48 \mathrm{~h}$ post infection (scale bar: $100 \mu \mathrm{m}$ ). Images are representative of two independent experiments. c Quantification of cytosolic and mitochondrial CypD following Mtb infection. MRC-5 lung fibroblasts were infected with Mtb (MOI 10), treated with dexamethasone $(5 \mu \mathrm{M})$, doramapimod $(10 \mu \mathrm{M})$, or CsA $(10 \mu \mathrm{M})$ and lysed for the isolation of mitochondria $5 \mathrm{~h}$ post infection. Equal amounts of protein from the mitochondrial and cytosolic fractions were subjected to western blot analysis and the levels of CypD were measured. Voltagedependent anion-selective channel 1 (VDAC-1) was used as a loading control. d Expression of hexokinase II (HKII) in Mtb- infected MRC-5 lung fibroblasts. Lysates were obtained $24 \mathrm{~h}$ after infection and were analyzed by qRT-PCR. e Quantificiation of mitochondrial HKII following Mtb infection. J774.2 M $\varphi$ were infected with $M t b$, treated with dexamethasone $(5 \mu \mathrm{M})$ or doramapimod $(10 \mu \mathrm{M})$, and mitochondria were isolated 5 and $24 \mathrm{~h}$ post infection. Equal amounts of protein were subjected to western blot analysis and the levels of HKII were measured. VDAC-1 was used as a loading control. $\mathbf{f}$ Quantification of HKII in the cytosol of MRC-5 lung fibroblasts. Whole-cell lysates were obtained from infected untreated cells and from infected cells treated with doramapimod (10 $\mu \mathrm{M}$ ) or BMS-582949 $(10 \mu \mathrm{M}) 48 \mathrm{~h}$ post infection. HKII was quantified by western blot analysis using $\beta$-actin as a loading control. Images are representative of two individual experiments. Representative data from at least two experiments with multiple replicates are shown in a and $\mathbf{d}$. Results are expressed as mean \pm SEM and experiments were analyzed using unpaired $t$-test (ns not significant; ${ }^{\star \star} p \leq 0.01 ;{ }^{\star \star \star} p \leq 0.001$ )

after Mtb infection (Fig. 5f). Mitochondrial HKII ensures tight coupling of glucose phosphorylation and ATP generation ${ }^{32}$. Parallel to mitochondrial dissociation of HKII, we observed a drastic decline of intracellular ATP levels after Mtb infection of different host cell types (Fig. 6a, b). This effect was reversed in dexamethasone and doramapimod treated cells (Fig. 6a, b). Cotreatment of $M t b$-infected $\mathrm{J} 774 \mathrm{M} \varphi$ with 3-bromopyruvate (3BP), an HKII inhibitor, abrogated the ATP-sparing effect of 

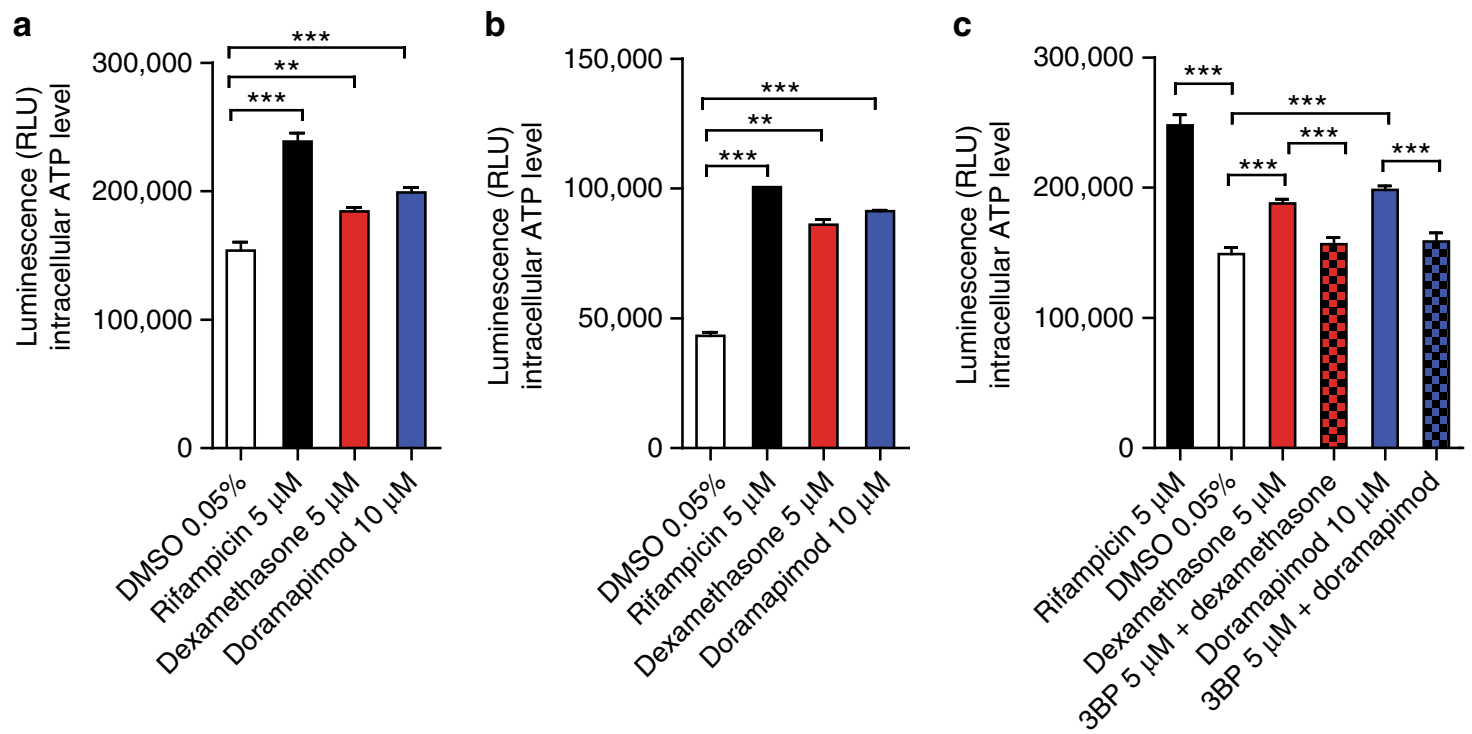

Fig. 6 p38 MAPK inhibition preserves intracellular ATP levels. a, b Intracellular ATP levels in Mtb-infected J774.2 M $(\mathbf{a})$ and MRC-5 lung fibroblasts (b) following 24 and $48 \mathrm{~h}$ of infection, respectively. Cells were treated with dexamethasone ( $5 \mu \mathrm{M})$ or doramapimod (10 $\mu$ M). c Intracellular ATP levels in Mtb-

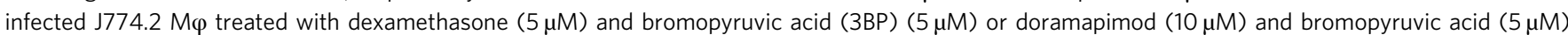
after $24 \mathrm{~h}$ of infection. Data from two experiments with multiple replicates are shown in $\mathbf{a}$ and $\mathbf{b}$ and data from one of two experiments are shown in c. Results are expressed as mean \pm SEM and experiments were analyzed using unpaired $t$-test $\left({ }^{\star \star} p \leq 0.01 ;{ }^{\star \star \star} p \leq 0.001\right)$

dexamethasone and doramapimod which links p38 MAPK activity to HKII (Fig. 6c).

The targeted p38 MAPK controlled pathway we identified seems to be specific for the intracellular pathogen $M t b$ since type III secretion system-mediated host cell death caused by Gram-negative pathogens, such as Pseudomonas aeruginosa and Salmonella typhimurium, could not be blocked under similar conditions (Fig. 7a, c). The same holds true for protection of macrophages infected with the intracellular pathogen Listeria monocytogenes (Fig. 7b). Though being potent inhibitors of $M t b$-induced necrosis, none of the described substances led to a change of the intracellular mycobacterial load of J774 $\mathrm{M} \varphi$ compared to untreated control cells (Supplementary Fig. 12). This elucidates that phagocytosis or intracellular replication of mycobacteria is not impaired by host cell pretreatment with the respective drugs. A finding which also explains why the potent necrosis inhibitors we identified were missed in other phagocyte-based drug screens using intracellular mycobacterial replication but not host cell survival as primary readout ${ }^{33}$.

Doramapimod treatment of mice is cytoprotective for infected M $\varphi$. To determine whether in vivo chemical p38 MAPK inhibition has the potential to reduce necrotic cell death in mouse $M \varphi$, we performed an experiment in which we treated C57BL/6 mice with doramapimod ( $100 \mathrm{mg} / \mathrm{kg}$ (q.d.; i.p.) for 3 days. The following day, $\mathrm{CD}_{11} \mathrm{~b}^{+}$peritoneal $\mathrm{M} \varphi$ were isolated using labeled magnetic microbeads and infected with $M t b$ at two different MOI. Twenty-four hours after infection, cell death was quantified by fluorescent staining. $M \varphi$ isolated from doramapimod-treated mice were strongly protected from $M t b$-induced cell death in contrast to cells from mice receiving the vehicle only (Fig. 8). This finding highlights the potential of chemical p38 MAPK inhibition as a therapeutic option to abrogate necrotic cell death caused by $M t b$ infection. In addition, the experiment rules out a direct inhibitory effect of doramapimod on $M t b$ since $\mathrm{M} \varphi$ infection was performed after in vivo treatment and extensive washing of $\mathrm{CD}_{11 \mathrm{~b}^{+}}$selected monocytes.

\section{Discussion}

Here, by exploiting a high-throughput chemical genetics approach, we show that corticosteroids possess a so far undescribed TNFa-independent inhibitory effect on necrotic cell death. Our detailed analysis revealed a highly druggable process involving the GR, MKP-1, and p38 MAPK, which ultimately impacts on $M t b$-induced necrosis by provoking mitochondrial MPT and loss of mitochondrial integrity (Fig. 9). Opening of the mPTP is an emerging mechanism of regulated necrosis which becomes increasingly associated with pathogenesis of several diseases $^{23}$. However, the exact molecular composition of the channel forming the MPTP is a matter of debate. Candidate molecules include the adenine nucleotide translocase, the voltagedependent anion channel (VDAC), and components of the ATP synthase $^{31}$. A confirmed regulatory component of the pore complex is CypD ${ }^{31}$. Accordingly, chemical CypD inhibition using CsA is a well-known mechanism to inhibit mPTP opening and has been previously shown to block $M t b$-induced cell death ${ }^{10,25}$. However, CsA is a non-selective cyclophylin inhibitor leading to immunosuppression by blocking calcineurin activity in $\mathrm{T}$ cells. Exploiting chemical mPTP inhibition as HDT requires either selective CypD inhibitors or substances that target alternative components of the complex. Here we showed that chemical activation of the GR or p38 MAPK inhibition does not impact on mitochondrial CypD but interferes with mitochondrial HKII function. HKII is primarily associated with the mitochondrial membrane where it binds VDAC and CypD ${ }^{32}$. The interaction of HKII with mitochondria represents an intrinsic molecular mechanism to protect cells against stress cues ultimately leading to necrotic death. Mitochondrial HKII has the ability to inhibit reactive oxygen species generation at mitochondria which prevents opening of the mPTP ${ }^{32,34}$. Subsequently, a decrease in HKII levels sensitizes cells to necrotic stimuli ${ }^{32}$. However, little is known on regulatory aspects of HKII transcription, posttranslational modification, and mitochondrial translocation. Here we provide indirect evidence that the activation of $\mathrm{p} 38$ MAPK by intracellular $M t b$ initiates mitochondrial dissociation of HKII, which, in turn, promotes cell death via mitochondrial permeability transition and ATP depletion. Interestingly, it was 
a

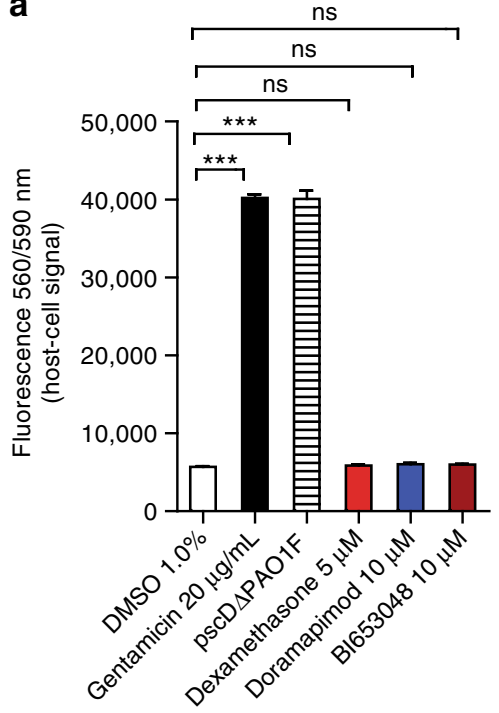

b

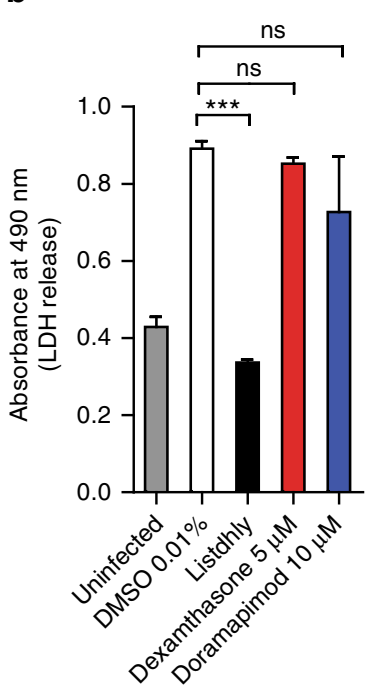

c

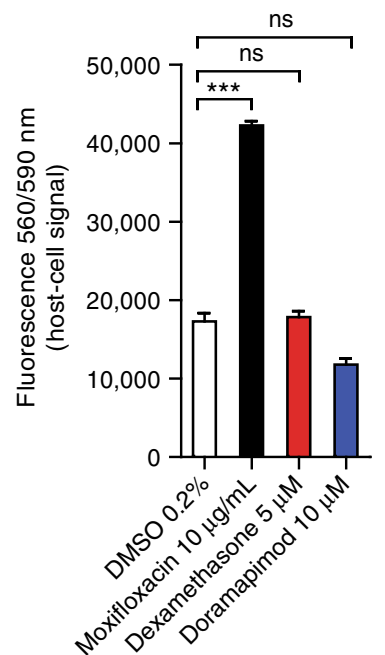

Fig. 7 p38 MAPK inhibition fails to protect host cells infected with other bacteria. a Effect of dexamethasone $(5 \mu \mathrm{M})$ and doramapimod (10 $\mu \mathrm{M})$ on Pseudomonas aeruginosa (strain PAO1F)-infected (MOI 0.5) A549 human lung epithelial carcinoma cells. Viability of A549 cells was quantified $24 \mathrm{~h}$ post infection using resazurin. A $\triangle \mathrm{pscC}$ mutant deficient in secretion of T3SS effector proteins was used as a control. $\mathbf{b}$ Dexamethasone $(5 \mu \mathrm{M})$ and doramapimod $(10 \mu \mathrm{M})$ have no cytoprotective effect in BMDM infected with Listeria monocytogenes (MOI 2). Host cell viability was quantified $20 \mathrm{~h}$ post infection by measuring LDH in the culture medium of the infected cells. A L. monocytogenes mutant strain (Listdhly) lacking the hly gene, which encodes the

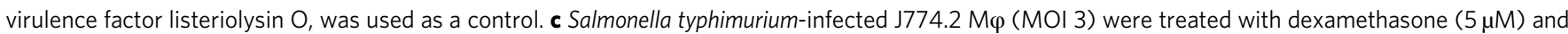
doramapimod $(10 \mu \mathrm{M})$. Host cell viability was quantified $48 \mathrm{~h}$ post infection using resazurin. Data from one of two experiments are shown in $\mathbf{a}$ and $\mathbf{c}$; data from two experiments with multiple replicates are shown in $\mathbf{b}$. Results are expressed as mean \pm SEM and experiments were analyzed using unpaired $t$-test (ns not significant; ${ }^{\star \star \star} p \leq 0.001$ )

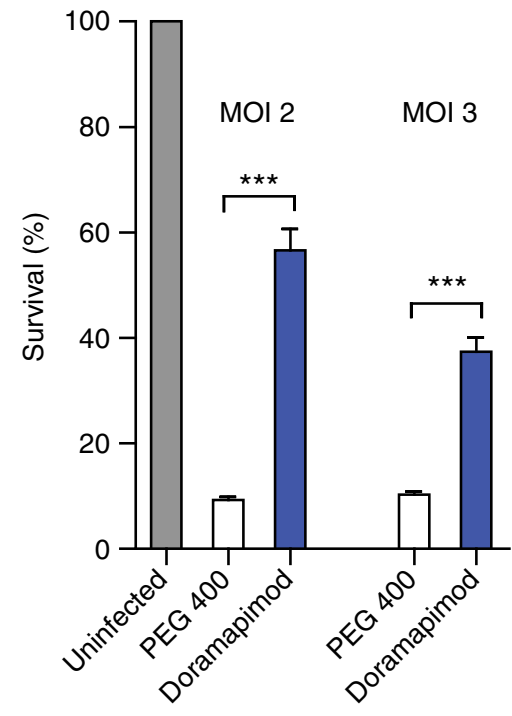

Fig. 8 Doramapimod treatment of mice is protective for peritoneal macrophages. Treatment of C57BL/6 mice with doramapimod is protective for $M t b$-infected peritoneal M $\varphi$ (PM). Mice received doramapimod (100 $\mathrm{mg} / \mathrm{kg}$ q.d.; i.p.) for 3 days. $\mathrm{PM}$ were isolated by $\mathrm{CD} 11 \mathrm{~b}^{+}$selection and infected with $\mathrm{Mtb}$ (MOI 2 or MOI 3). Cell survival was assessed $24 \mathrm{~h}$ after infection by DAPI staining, using uninfected PM as a control. Control mice received the vehicle PEG 400. Data from one experiment with three mice per group and multiple replicates are shown. Results are expressed as mean \pm SEM and experiments were analyzed using unpaired $t$-test $\left({ }^{\star \star \star} p \leq 0.001\right)$

recently shown that pathogen-driven HKII dissociation represents an important means of immune sensing in other Grampositive bacteria ${ }^{35}$. Thus, HKII seems to be a common bacterial target in host-pathogen interactions of Gram-positive organisms, although the underlying molecular mechanisms still need to be determined. Our data showing that in $M t b$-infected cells, HKII dissociation can be abrogated by MKP-1 activation (dexamethasone, BI653048) and p38 MAPK inhibition represent a possible molecular link to further elucidate the regulatory mechanisms of HKII.

p38 MAPK is a validated target in autoimmune and inflammatory diseases and several small-molecule inhibitors have been tested in clinical trials mainly against rheumatoid arthritis, psoriasis, and Crohn's disease ${ }^{36}$. Histopathological investigation of human biopsies revealed p38 MAPK phosphorylation in $M \varphi$ surrounding TB-granulomas, indicating that this kinase is engaged and may be a potential target for $\mathrm{HDT}$ in $\mathrm{TB}^{37}$. We were surprised to identify differing activity of several commercially available and clinically tested p38 MAPK inhibitors. All tested substances are highly specific for p38 MAPK and display an $\mathrm{IC}_{50}$ in the nanomolar range ${ }^{36}$. However, among the tested substances there are differences with regard to inhibition of the p38 MAPK isotypes $\alpha, \beta, \gamma$, and $\delta$. Doramapimod is a broad spectrum inhibitor potently targeting all four isotypes whereas pamapimod is much more selective for the $\alpha$ and $\beta$ isotypes. In addition, the reason for doramapimod being the sole inhibitor that maintained low phosphorylation levels of p38 MAPK at later time points of infection may also be found in its unique binding mechanism on the target kinas $\mathrm{e}^{38}$. Our data showing protection of $\mathrm{M} \varphi$ after $\mathrm{p} 38$ MAPK gene knock down confirm dephosphorylation and activity data obtained using doramapimod and verify the key role of this kinase in $M t b$-induced cell death.

It is unlikely that p38 MAPK directly interacts with mitochondrial bound HKII and identification of the linking molecule requires further research. A starting point could be p53, a regulatory protein which shuttles between different cell compartments and physically interacts with the mitochondrial membrane ${ }^{39}$. We have generated preliminary data indicating that 


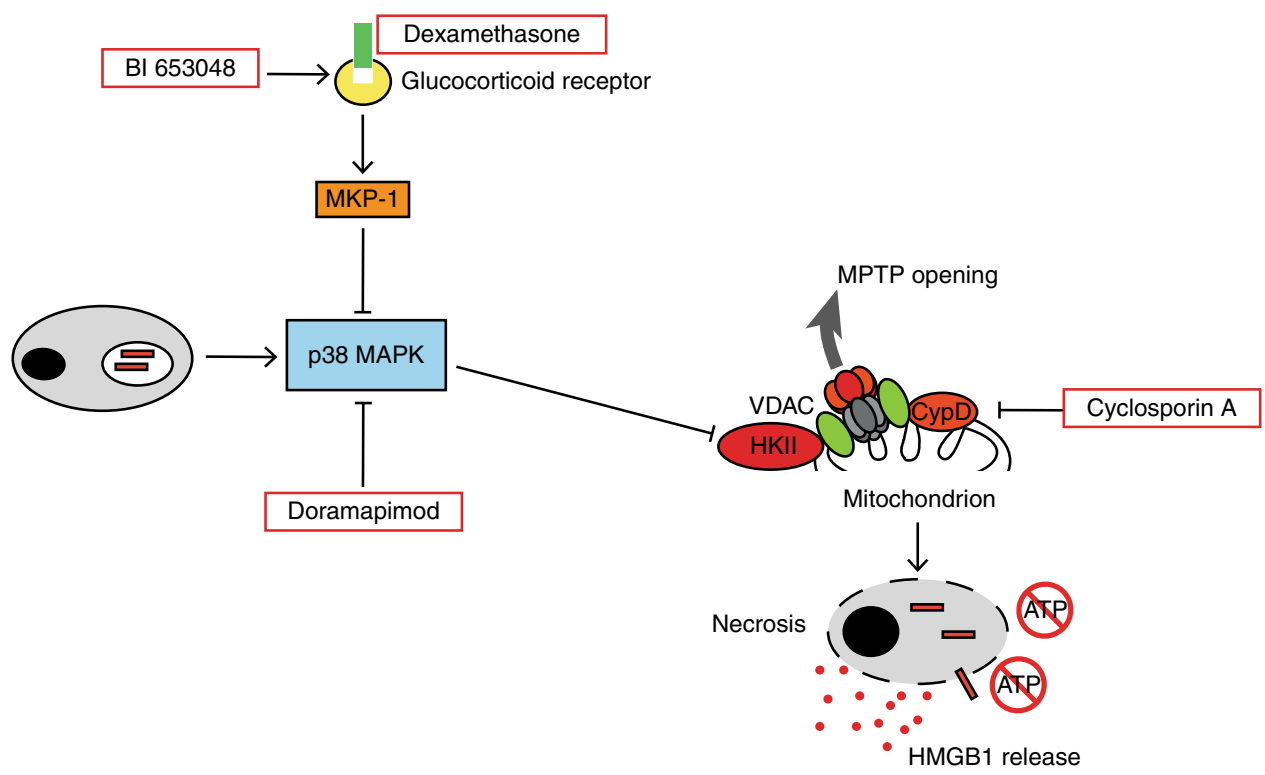

Fig. 9 Model depicting a novel mode of Mtb-induced cell death and its chemical inhibition. Phagocytosis of Mtb leads to p38 MAPK phosphorylation and hexokinase II (HKII) dissociation, which seems to open the mitochondrial permeability transition pore (mPTP) subsequently leading to necrotic cell death which can be monitored by high-mobility group protein B1 (HMGB1) release. Several substances interfere with this pathway to protect cells.

Dexamethasone or B1653048 treatment inhibits p38 MAPK phosphorylation via the glucocorticoid receptor (GR) and MAPK phosphatase 1 (MKP-1). Direct p38 MAPK blockade using doramapimod has a similar cytoprotective effect. p38 MAPK promotes dissociation of hexokinase II (HKII) from the mitochondrion leading to ATP depletion. Cyclosporin A directly interferes with mPTP opening via inhibition of cyclophillin $D$, a regulator of the mPTP. VDAC voltage-dependent anion channel

chemical p53 inhibition in $M t b$-infected cells has similar effects as p38 MAPK inhibition with regard to cytoprotection and ATP depletion (Supplementary Fig. 13). In the past decade, numerous reports have highlighted the crucial role of $\mathrm{p} 53$ in host-pathogen interactions ${ }^{40}$. p5 53 is known to influence mPTP either via its own mitochondrial translocation or by indirect modulation of mPTP components including $\mathrm{HKII}^{39,41-43}$. In addition, p38 MAPKmediated p53 phosphorylation constitutes a critical step in pathogen-driven cell death and chemotherapeutic killing of cancer cells ${ }^{44,45}$. This possible link in $M t b$ pathogenesis requires a more detailed examination in the future.

By using genetic and chemical targeting, we provide clear evidence that TNFa and RIPK1/MLKL-driven necroptosis is not involved in corticosteroid- and p38 MAPK-dependent mitochondrial depolarization in $M t b$-infected cells. Two studies linked RIP-kinase signaling to mitochondrial membrane depolarization and cell death in $\mathrm{TB}^{10,25}$. Our contradictory findings confirm and further extend results of a recent study on $\mathrm{Mlkl}^{-1-}$ mice which presented lung histopathology and mycobacterial burdens indistinguishable from control mice ${ }^{15}$. Collectively, these data show that necroptotic signaling is unlikely a trigger for MPTP opening in TB. In addition, our findings on TNFa-independent hostprotective effects of corticosteroids may also explain clinical data showing that adjuvant corticosteroid treatment is more beneficial for TB patients than adjuvant treatment with specific TNFa inhibitors ${ }^{46}$. Our findings have immediate consequences for future HDT trials targeting primarily TNFa.

In summary, our report indicates that $M t b$ infection leads to phosphorylation of p38 MAPK and mitochondrial HKII dissociation, which mediates cell death via mitochondrial permeability transition and ATP depletion. A mechanism which can be disrupted by MKP-1 activation (dexamethasone, BI653048) and p38 MAPK inhibition (Fig. 9). These findings may have the potential to extend the portfolio of host-directed anti-TB therapeutics. Finally, we provide proof of concept for readout switching from bacterial growth inhibition to host cell survival, which can identify inhibitors of $M t b$-induced necrotic host cell death in future high-throughput drug screens.

\section{Methods}

Ethics statement. All animal experiments were performed in accordance with institutional, state, and federal guidelines (approved by the Landesamt fuer Natur, Umwelt und Verbraucherschutz (LANUV) North Rhine-Westphalia, Germany).

Blood samples were obtained from patients with active TB before therapy was initiated and from healthy volunteers. The study was approved by the University of Cologne Ethics Committee (18-079). Patients as well as healthy volunteers participated after giving written informed consent.

Drugs used in this study. Rifampicin was purchased from AppliChem. Bromopyruvic acid, dexamethasone, gentamicin, staurosporine, and thalidomide were purchased from Sigma-Aldrich. Cyclosporin A, doramapimod, moxifloxacin, necrostatin-1, necrostatin-5, and tacrolimus (FK506) were obtained from Cayman Chemicals. BMS-582949, pamapimod, and Z-VAD-FMK were purchased from Selleckchem. Puromycin dihydrochloride was from Carl Roth (Germany). GR agonist BI653048 was provided by the opnMe platform (Boehringer Ingelheim). The Prestwick chemical library was used for high-throughput screening.

Mice. Six- to eight-week-old female C57BL/6 mice were bred in-house and used at an age of 8-12 weeks. Animals were housed under specific pathogen-free conditions in the Laboratory Animal Facility of the University Hospital Cologne.

Isolation of human macrophages. Human mononuclear leukocytes were isolated from the blood of healthy donors or TB patients and monocytes were obtained by magnetic-activated cell sorting (MACS) of CD14 ${ }^{+}$cells using CD14 MicroBeads (Miltenyi Biotec). Monocytes were differentiated into $\mathrm{M} \varphi$ for 4 days in Roswell Park Memorial Institute 1640 (RPMI) medium supplemented with 10\% fetal bovine serum (FBS) and $50 \mathrm{ng} / \mathrm{ml}$ human macrophage colony-stimulating factor (Miltenyi Biotec) at a density of $1 \times 10^{5}$ cells per well in 96-well plates (Corning). Before infecting primary $\mathrm{M} \varphi$ with varying MOI ( 1 or 2 ) of $M t b$, the growth media was changed to RPMI supplemented with $10 \% \mathrm{FBS}$. M $\varphi$ were grown at $37^{\circ} \mathrm{C}$ with $5 \% \mathrm{CO}_{2}$

Isolation of mouse macrophages. Bone-marrow-derived $\mathrm{M} \varphi$ (BMDM) were isolated as previously described ${ }^{47}$. Briefly, mice were sacrificed by cervical dislocation, the legs were removed, and dissected from adherent tissue. Femurs and 
tibia were cut off at both ends and the bone marrow was expelled with RPMI using a $27 \mathrm{~g}$ needle. After centrifugation for $10 \mathrm{~min}$ at $400 \times g$ cells were plated in Petri dishes and incubated at $37^{\circ} \mathrm{C}$ and $5 \% \mathrm{CO}_{2}$. Bone marrow cells were differentiated in VLE RPMI 1640 (Biochrom) supplemented with 10\% FCS (Biowest), $10 \mathrm{mM}$ HEPES (Biochrom), $10 \mu \mathrm{g} / \mathrm{ml}$ penicillin/streptomycin (Biochrom), $1 \mathrm{mM}$ sodium pyruvate (Biochrom), 2mM L-glutamine (Biochrom), and 15\% M-CSF (supernatants of L929 mouse fibroblasts), for 7 days. Five days after start of the culture fresh medium was added.

Peritoneal $\mathrm{M} \varphi(\mathrm{PM})$ were isolated from C57BL/6 mice by peritoneal lavage and subsequent $\mathrm{CD} 11 \mathrm{~b}^{+}$selection by MACS using CD11b MicroBeads (Miltenyi Biotec). For ex vivo experiments, mice received doramapimod $(100 \mathrm{mg} / \mathrm{kg}$, in polyethylene glycol 400 (PEG 400) i.p.) for 3 days and control mice received PEG 400 only. PM were isolated and seeded in 96 -well plates at a density of $5 \times 10^{4}$ cells per well in Dulbecco's modified Eagle's medium (DMEM) supplemented with 10\% FBS. Following adherence of the cells, PM were infected with Mtb (MOI 2 or 3) for $24 \mathrm{~h}$ and analyzed by fluorescence microscopy.

Cell culture. MRC-5 human lung fibroblasts were provided by the Coriell Institute for Medical Research and cultured in minimum essential medium (MEM) supplemented with $10 \%$ heat-inactivated FBS(PAN-Biotech), $1 \%$ non-essential amino acids, and $1 \%$ sodium pyruvate. J774A.1 $\mathrm{M} \varphi$ and $7774.2 \mathrm{M} \varphi$ (ATCC) were grown in DMEM supplemented with 10\% FBS. BV-2 microglia were grown in DMEM supplemented with $10 \%$ FBS and $1 \%$ sodium pyruvate. A549 human lung epithelial carcinoma cells (ATCC) were grown in RPMI medium supplemented with $10 \%$ FBS.

Culture conditions of bacteria. The mycobacterial strain Erdman (provided by S.T. Cole) was grown in Middlebrook $7 \mathrm{H} 9$ broth, supplemented with $10 \%$ albumin dextrose catalase (ADC), $0.05 \%$ Tween- 80 and $0.2 \%$ glycerol, or $7 \mathrm{H} 10$ agar plates, supplemented with $10 \%$ ADC and $0.5 \%$ glycerol. All P. aeruginosa strains (PAO1F WT and PAO1F $\triangle \mathrm{pscD}$ ) were routinely grown in $2 \mathrm{xYT}$ medium and all $S$. typhimurium strains as well as L. monocytogenes strains were grown in brain heart infusion medium.

Survival assays. For the survival assays, compounds were preplated into 96-well plates at different concentrations. MRC-5, J774.2 M $\varphi$, or A549 cells were harvested and seeded at a density of $2 \times 10^{4}$ cells per well and were allowed to adhere for $3 \mathrm{~h}$. Then cells were infected with $M t b$ Erdman with varying MOI

For high-throughput screening, MRC-5 lung fibroblasts were infected with an MOI of 10 and were analyzed $72 \mathrm{~h}$ post infection using the fibroblast survival assay as previously described ${ }^{19,20}$. In brief, 4000 MRC- 5 cells were seeded in 384 -well cell bind plates (Corning). After $3 \mathrm{~h}$ at $37^{\circ} \mathrm{C}$, cells were infected with $M t b$ Erdman resuspended in MEM medium. After the $72 \mathrm{~h}$ incubation period, survival of fibroblasts was determined by addition of PrestoBlue ( $10 \%$ final concentration) and fluorescence reading in a Cytation 3 Cell Imaging Multi-Mode Reader (Biotek).

For the survival assay with $P$. aeruginosa, A549 cells were seeded in a 96- well plate containing different drugs. Following adherence, cells were infected with $P$. aeruginosa at an MOI of 0.5 and after $4 \mathrm{~h}$ moxifloxacin was added at a final concentration of $10 \mu \mathrm{M}$. The next day, $10 \%$ resazurin was added and fluorescence was detected using a Tecan Safire II fluorescence reader.

For the survival assay with S. typhimurium, J774.2 M $\varphi$ were infected at an MOI of 2 with $S$. typhimurium for $3 \mathrm{~h}$, before adding moxifloxacin at a final concentration of $10 \mu \mathrm{M}$. After $48 \mathrm{~h}$ of incubation, $10 \%$ resazurin was added and fluorescence was detected using a Tecan Safire II fluorescence reader.

For the survival assay with L. monocytogenes, BMDM were seeded at a density of $8 \times 10^{4}$ cells per well in a 96-well plate and differentiated into macrophages for 7 days ${ }^{47}$. BMDM were then infected with L. monocytogenes at an MOI of 2 for $2 \mathrm{~h}$, before adding moxifloxacin at a final concentration of $10 \mu \mathrm{M}$. The following day, LDH was measured in the supernatant of the cells.

Fluorescence microscopy. For fluorescence microscopy J774 M $\varphi$ and BV-2 microglia were seeded at a density of $2 \times 10^{4}$ cells per well in a 96-well plate. Following adherence, cells were infected with $M t b$ Erdman at an MOI of 5 for $48 \mathrm{~h}$. Isolated primary human monocytes were seeded at a density of $1 \times 10^{5}$ cells per well and BMDM were seeded at a density of $8 \times 10^{4}$ cells per well in a 96-well plate. After differentiation of monocytes into $\mathrm{M} \varphi$, cells were infected with $M t b$ for $48 \mathrm{~h}$. PM were seeded at a density of $5 \times 10^{4}$ cells per well in a 96-well plate and infected with $M t b$ at an MOI of 2 or 3 for $24 \mathrm{~h}$. Afterwards, cells were washed several times with PBS and fixed with $4 \%$ paraformaldehyde/PBS. To quantify the number of surviving cells, cells were stained with 4',6-diamidino-2-phenylindole (DAPI; Thermo Fisher Scientific) and images were acquired on an IX81 inverted microscope (Olympus) using cellSens standard software (Olympus) as well as Fiji processing software.

CFU determination. J774.2 $\mathrm{M} \varphi$ were seeded one day prior to the infection in 96well plates at a density of $5 \times 10^{3}$ cells per well. Cells were pre-treated with different drugs for $2 \mathrm{~h}$ and infected with $M t b$ Erdman at an MOI of 2. The following day, cells were washed several times with PBS to remove unphagocytosed bacteria and fresh medium containing compounds or DMSO was added. Plates were sealed and incubated at $37^{\circ} \mathrm{C}$ in $5 \% \mathrm{CO}_{2}$. Five days post infection cells were lysed with $0.1 \%$ sodium dodecyl sulfate (SDS). Viable bacteria were grown in serial dilutions on $7 \mathrm{H} 10$ agar plates. Colonies were counted after $10-14$ days of incubation at $37^{\circ} \mathrm{C}$.

HMGB1 ELISA. The release of HMGB1 from cells was detected by using a HMGB1 ELISA kit (IBL International). Briefly, $1 \times 10^{6} \mathrm{MRC}-5$ lung fibroblasts were seeded in a six-well plate and the following day cells were infected with $M t b$ Erdman at an MOI of 10. Infected cells were incubated for $48 \mathrm{~h}$ at $37^{\circ} \mathrm{C}$ in $5 \% \mathrm{CO}_{2}$ and the supernatant was collected. The samples were transferred into a 96 -well plate precoated with polyclonal anti-HMGB1 and incubated for $24 \mathrm{~h}$ at $37^{\circ} \mathrm{C}$. Afterwards, HMGB1 conjugated to peroxidase was added to the samples and the plate was incubated for another $2 \mathrm{~h}$ at $25^{\circ} \mathrm{C}$. For detection, TMB substrate solution was used. The absorbance was measured and analyzed using a Multiskan ${ }^{\mathrm{su}}$ FC Microplate Photometer with internal software (Thermo Fisher Scientific).

Caspase assay. Caspase activity was measured with the Caspase-Glo ${ }^{\bullet}$ 3/7 Assay according to the manufacturer's instructions (Promega). Cells were harvested and seeded at a density of $2 \times 10^{4}$ cells per well in a 96-well plate. Following adherence of the cells, MRC-5 lung fibroblasts (MOI 10) and J774.2 M $\varphi$ (MOI 5) were infected with $M t b$ Erdman and incubated for up to $48 \mathrm{~h}$ at $37^{\circ} \mathrm{C}$ in $5 \% \mathrm{CO}_{2}$. The Caspase- Glo Reagent was added to the wells 24 and $48 \mathrm{~h}$ post infection and luminescence was measured in a BioTek ${ }^{\text {mo }}$ Cytation $^{\text {me }} 3$ Cell Imaging Multi-Mode Reader.

LDH release assay. The Pierce ${ }^{\mathrm{Tu}}$ LDH Cytotoxicity Assay Kit (Thermo Fisher Scientific) was conducted according to the manufacturer's recommendations. Harvested MRC-5 lung fibroblasts and J774.2 $\mathrm{M} \varphi$ were seeded in a 96-well plate $\left(2 \times 10^{4}\right.$ cells per well) and infected with Mtb Erdman at an MOI of 10 and 5, respectively. Twenty-four, 48 and $72 \mathrm{~h}$ post infection the release of LDH into the supernatant of cells was analyzed by measuring the reduction of tetrazolium salt to a red formazan product in a BioTek ${ }^{\text {Tw }}$ Cytation $^{\text {sw }} 3$ Cell Imaging Multi-Mode Reader (Biotek). The reaction mixture was added to the samples at a ratio of 1:1 and the plates were incubated for $30 \mathrm{~min}$ at room temperature. Lysed cells were used as maximum LDH activity control and untreated cells were used as spontaneous LDH release control. For primary human $M \varphi, 1 \times 10^{5}$ cells per well were infected with an MOI of 1 or 2 with $M t b$ and the supernatant was collected 24 and $48 \mathrm{~h}$ post infection to measure the release of $\mathrm{LDH}$.

ATP assay. The CellTiter-Glo ${ }^{\circ} 2.0$ Assay (Promega) was conducted according to the manufacturer's recommendations. Harvested MRC-5 lung fibroblasts and J774.2 $\mathrm{M} \varphi$ were seeded in a 96-well plate $\left(2 \times 10^{4}\right.$ cells per well $)$ and infected with $M t b$ Erdman at an MOI of 10 and 5, respectively. Twenty-four and $48 \mathrm{~h}$ post infection the amount of ATP was quantified by adding the CellTiter-Glo 2.0 reagent to the samples at a ratio of 1:1 and incubating the plate for $10 \mathrm{~min}$ at room temperature. Afterwards luminescence was detected in a BioTek ${ }^{\text {mit }}$ Cytation $^{\text {tw }} 3$ Cell Imaging Multi-Mode Reader.

Isolation of mitochondria. For the isolation of mitochondria from infected MRC5 lung fibroblasts (MOI 10) and J774.2 $\mathrm{M} \varphi$ (MOI 5) the Mitochondria Isolation Kit for Cultured Cells (Thermo Fisher Scientific) was used according to the manufacturer's recommendations. Briefly, 5 and $24 \mathrm{~h}$ post infection $1 \times 10^{7}$ cells were washed several times with PBS and detached from the culture dish using a cell scraper. Mitochondria were obtained using the reagent-based method. For separation of the mitochondrial and the cytosolic fraction, the sample was centrifuged at $12,000 \times g$ for $15 \mathrm{~min}$ at $4^{\circ} \mathrm{C}$. The mitochondria were lysed with $2 \%$ CHAPS (Thermo Fisher Scientific) in Tris-buffered saline (TBS; $25 \mathrm{mM}$ Tris, $150 \mathrm{mM} \mathrm{NaCl}, \mathrm{pH}$ 7.2) containing Halt ${ }^{\mathrm{mt}}$ Protease and Phosphatase Inhibitor Cocktail (Thermo Fisher Scientific). Both the mitochondrial and cytosolic fractions were stored at $-80^{\circ} \mathrm{C}$ and the purity of the mitochondrial fraction was analyzed by western blotting.

TMRM staining. J774.2 M $\varphi$ were seeded in a 96 -well plate $\left(5 \times 10^{3}\right.$ per well $)$ and infected with $M t b$ Erdman at an MOI of 5 for varying times ( 24 and $48 \mathrm{~h}$ ). For staining with TMRM, cells were washed several times with PBS before adding a final concentration of $100 \mathrm{nM}$ of TMRM (Sigma-Aldrich). Cells were incubated at $37^{\circ} \mathrm{C}$ in $5 \% \mathrm{CO}_{2}$ for $30 \mathrm{~min}$ and washed with PBS. Images were acquired on an IX81 inverted microscope (Olympus) using cellSens standard software (Olympus) as well as Fiji processing software.

Immunoblot analysis. $1 \times 10^{6}$ MRC-5 lung fibroblasts or $\mathrm{J} 774.2 \mathrm{M} \varphi$ were seeded in $10 \mathrm{~cm}$ culture dishes and allowed to adhere for at least $24 \mathrm{~h}$ before infection Whole-cell lysates were obtained 5, 24, 30, and $48 \mathrm{~h}$ post infection using radioimmunoprecipitation assay (RIPA) buffer. The protein concentration was measured with the Pierce ${ }^{\mathrm{mx}}$ BCA Protein Assay Kit (Thermo Fisher Scientific). Proteins were separated by SDS-polyacrylamide gel electrophoresis and transferred to a PVDF membrane using the Trans-Blot ${ }^{6}$ Turbo $^{\text {mex }}$ Transfer System (Bio Rad). The membrane was blocked for $1 \mathrm{~h}$ with either 5\% dried milk for total proteins or 5\% bovine serum albumin for phosphorylated proteins in Tris- $\mathrm{HCl}$ buffer $(10 \mathrm{mM}, \mathrm{pH}$ 
7.6), containing $\mathrm{NaCl}(150 \mathrm{mM})$ and Tween 20 (1\%). Subsequently, the membrane was incubated with primary antibodies at $4{ }^{\circ} \mathrm{C}$ overnight. The next day, the blots were incubated for $1 \mathrm{~h}$ at room temperature with the corresponding secondary, horseradish peroxidase-conjugated, antibody. Visualization of the transferred proteins was done by using the 20X LumiGLO ${ }^{\circ}$ Reagent and 20X Peroxide (Cell Signaling Technology) and X-ray films were processed in a Curix 60 (AGFA). All antibodies except anti-cyclophilin D (ab110324; Abcam) were purchased from Cell Signaling Technology and used according to the manufracturer's recommendations. The following antibodies were used: anti- $\beta$-Aktin (4970), anti-p38 MAPK (9212), anti-phospho p38 MAPK (4511), anti-JNK (9252), anti-phospho JNK (4688), anti-ERK (9102), anti-phospho ERK (9101), anti- caspase 3 (9662), anti-cleaved caspase 3 (9661), anti-hexokinase II (2867), and anti-VDAC-1 (4866).

Quantitative real-time PCR. For the isolation of total RNA, the RNeasy Mini Kit (Qiagen) was used. Briefly, $1 \times 10^{6}$ MRC-5 lung fibroblasts and J774.2 M $\varphi$ were seeded in a six-well plate and infected with Mtb Erdman at an MOI of 10 and 5, respectively. Five and $24 \mathrm{~h}$ post infection, cells were washed several times with PBS, detached from the plate by using a cell scraper, and lysed with RLT buffer containing $\beta$-mercaptoethanol. Quantification of RNA was done using a NanoDrop spectrophotometer (Thermo Fisher Scientific); $A_{260} / A_{280}$ ratios of all samples remained within the range of 1.90-2.10. cDNA synthesis was performed with the SuperScript III First-Strand Synthesis SuperMix for qRT-PCR (Thermo Fisher Scientific) and real-time PCR was conducted using Light Cycler Fast Start DNA MasterPLUS SYBR Green Kit (Roche) and specific primers for hexokinase II (fw 5'-TCTAAGCGGTTCCGCA; rev 5'-AGAAGGGTCATACCTGG), MKP-1 (fw 5'GGAATCTGGGTGCAGT; rev 5'-CTGGTAGTGACCCTCAA), p38 MAPK (fw 5'-GCCCGAACGATACCAG; rev 5'-CTGAAACGGTCTCGACA), GAPDH (fw 5'-GGTATCGTGGAAGGACT; rev 5'-GGGTGTCGCTGTTGAA), and HMBS ( $\mathrm{fw}$ $5^{\prime}$-TGCACGATCCCGAGAC; rev 5'-CGTGGAATGTTACGAGC). The analysis was done by comparative $\mathrm{Cp}$ method and $\mathrm{Cp}$ values were normalized against the house-keeping genes GAPDH and HMBS. To eliminate non-specific amplification, a melting curve analysis was performed.

Generation of a p38 knock-down macrophage cell line. Utilizing the retroviral MSCV-LTRmiR30-PIG (MLP) vector ${ }^{48}$ a p38-specific shRNA based on predictions from siRNA Scales ${ }^{49}$ and RNAi central (http://katahdin.cshl.edu) (Sequence: TGCTGTTGACAGTGAGCGATACCACGATCCTGATGATGAATAGTGAAG CCACAGATGTATTCATCATCAGGATCGTGGTACTGCCTACTGCCTCGGA) was generated. J774A.1 cells were transduced with shP38-MLP and MLP control vector respectively by puromycin selection. Knock-down efficiency was determined by qRT-PCR as described above.

Statistical analysis and general methods. Data are expressed as mean \pm SD or mean \pm SEM. The data were analyzed with the Graphpad Prism version 7 software program. Differences were assessed using two-tailed Student t-tests and differences with $p$ values of $<0.05$ were considered to be statistically significant. Sample size for animal studies was chosen based on prior experience with similar models and published data showing similar experiments. No data were excluded from the analysis of experiments. Mice were commercially sourced and randomized into experimental groups upon arrival, and all animals within a single experiment were processed at the same time, there was no blinding performed. Data display similar variance between groups and are normally distributed where parametric tests have been used.

Reporting Summary. Further information on experimental design is available in the Nature Research Reporting Summary linked to this article.

\section{Data availability}

The authors declare that all data supporting the findings of this study are available within the paper and its supplementary information files.

Received: 7 May 2018 Accepted: 9 January 2019

Published online: 08 February 2019

\section{References}

1. WHO. Global tuberculosis report (WHO, Geneva, 2017).

2. Korbee, C. J. et al. Combined chemical genetics and data-driven bioinformatics approach identifies receptor tyrosine kinase inhibitors as hostdirected antimicrobials. Nat. Commun. 9, 358 (2018).

3. Kaufmann, S. H. E., Dorhoi, A., Hotchkiss, R. S. \& Bartenschlager, R. Hostdirected therapies for bacterial and viral infections. Nat. Rev. Drug. Discov. 17, 35-56 (2018).

4. Wallis, R. S. \& Hafner, R. Advancing host-directed therapy for tuberculosis. Nat. Rev. Immunol. 15, 255-263 (2015).
5. Guler, R. \& Brombacher, F. Host-directed drug therapy for tuberculosis. Nat Chem. Biol. 11, 748-751 (2015).

6. Wallis, R. S. \& Zumla, A. Vitamin D as adjunctive host-directed therapy in tuberculosis: a systematic review. Open Forum Infect. Dis. 3, ofw151 (2016).

7. Aguilo, N., Uranga, S., Marinova, D., Martin, C. \& Pardo, J. Bim is a crucial regulator of apoptosis induced by Mycobacterium tuberculosis. Cell Death Dis. 5, e1343 (2014).

8. Derrick, S. C. \& Morris, S. L. The ESAT6 protein of Mycobacterium tuberculosis induces apoptosis of macrophages by activating caspase expression. Cell Microbiol. 9, 1547-1555 (2007).

9. Lee, J., Remold, H. G., Ieong, M. H. \& Kornfeld, H. Macrophage apoptosis in response to high intracellular burden of Mycobacterium tuberculosis is mediated by a novel caspase-independent pathway. J. Immunol. 176, 4267-4274 (2006)

10. Roca, F. J. \& Ramakrishnan, L. TNF dually mediates resistance and susceptibility to mycobacteria via mitochondrial reactive oxygen species. Cell 153, 521-534 (2013).

11. Tobin, D. M. et al. The lta4h locus modulates susceptibility to mycobacterial infection in zebrafish and humans. Cell 140, 717-730 (2010).

12. Tobin, D. M. et al. Host genotype-specific therapies can optimize the inflammatory response to mycobacterial infections. Cell 148, 434-446 (2012).

13. Curtis, J. et al. Association analysis of the LTA4H gene polymorphisms and pulmonary tuberculosis in 9115 subjects. Tuberculosis (Edinb.) 91, 22-25 (2011).

14. van Laarhoven, A. et al. Clinical parameters, routine inflammatory markers, and LTA4H genotype as predictors of mortality among 608 patients with tuberculous meningitis in Indonesia. J. Infect. Dis. 215, 1029-1039 (2017).

15. Stutz, M. D. et al. Necroptotic signaling is primed in Mycobacterium tuberculosis-infected macrophages, but its pathophysiological consequence in disease is restricted. Cell Death Differ 25, 951-965 (2018).

16. Prasad, K., Singh, M. B. \& Ryan, H. Corticosteroids for managing tuberculous meningitis. Cochrane Database Syst. Rev. 4, CD002244 (2016).

17. Thwaites, G. E. et al. Dexamethasone for the treatment of tuberculous meningitis in adolescents and adults. N. Engl. J. Med. 351, 1741-1751 (2004).

18. Hsu, T. et al. The primary mechanism of attenuation of bacillus CalmetteGuerin is a loss of secreted lytic function required for invasion of lung interstitial tissue. Proc. Natl. Acad. Sci. USA 100, 12420-12425 (2003).

19. Rybniker, J. et al. Anticytolytic screen identifies inhibitors of mycobacterial virulence protein secretion. Cell Host Microbe 16, 538-548 (2014).

20. Rybniker, J. et al. Lansoprazole is an antituberculous prodrug targeting cytochrome bcl. Nat. Commun. 6, 7659 (2015).

21. Abraham, S. M. et al. Antiinflammatory effects of dexamethasone are partly dependent on induction of dual specificity phosphatase 1. J. Exp. Med. 203, 1883-1889 (2006)

22. Caunt, C. J. \& Keyse, S. M. Dual-specificity MAP kinase phosphatases (MKPs): shaping the outcome of MAP kinase signalling. FEBS J. 280, 489-504 (2013).

23. Vanden Berghe, T., Linkermann, A., Jouan-Lanhouet, S., Walczak, H. \& Vandenabeele, P. Regulated necrosis: the expanding network of non-apoptotic cell death pathways. Nat. Rev. Mol. Cell Biol. 15, 135-147 (2014).

24. Butler, R. E. et al. Susceptibility of Mycobacterium tuberculosis-infected host cells to phospho-MLKL driven necroptosis is dependent on cell type and presence of TNFalpha. Virulence 8, 1820-1832 (2017).

25. Zhao, X. et al. Bcl-xL mediates RIPK3-dependent necrosis in M. tuberculosisinfected macrophages. Mucosal Immunol. 10, 1553-1568 (2017).

26. Dannappel, M. et al. RIPK1 maintains epithelial homeostasis by inhibiting apoptosis and necroptosis. Nature 513, 90-94 (2014).

27. Witt, A. et al. IAP antagonization promotes inflammatory destruction of vascular endothelium. EMBO Rep. 16, 719-727 (2015).

28. Linkermann, A. \& Green, D. R. Necroptosis. N. Engl. J. Med. 370, 455-465 (2014).

29. Vercammen, D. et al. Inhibition of caspases increases the sensitivity of L929 cells to necrosis mediated by tumor necrosis factor. J. Exp. Med. 187, 1477-1485 (1998).

30. Zhang, D. W. et al. RIP3, an energy metabolism regulator that switches TNFinduced cell death from apoptosis to necrosis. Science 325, 332-336 (2009).

31. Biasutto, L., Azzolini, M., Szabo, I. \& Zoratti, M. The mitochondrial permeability transition pore in AD 2016: an update. Biochim. Biophys. Acta 1863, 2515-2530 (2016).

32. Roberts, D. J. \& Miyamoto, S. Hexokinase II integrates energy metabolism and cellular protection: Akting on mitochondria and TORCing to autophagy. Cell Death Differ. 22, 364 (2015).

33. Christophe, T. et al. High content screening identifies decaprenylphosphoribose $2^{\prime}$ epimerase as a target for intracellular antimycobacterial inhibitors. PLoS Pathog. 5, e1000645 (2009).

34. da-Silva, W. S. et al. Mitochondrial bound hexokinase activity as a preventive antioxidant defense: steady-state ADP formation as a regulatory mechanism of membrane potential and reactive oxygen species generation in mitochondria. J. Biol. Chem. 279, 39846-39855 (2004).

35. Wolf, A. J. et al. Hexokinase is an innate immune receptor for the detection of bacterial peptidoglycan. Cell 166, 624-636 (2016). 
36. Arthur, J. S. \& Ley, S. C. Mitogen-activated protein kinases in innate immunity. Nat. Rev. Immunol. 13, 679-692 (2013).

37. Rand, L., Green, J. A., Saraiva, L., Friedland, J. S. \& Elkington, P. T. Matrix metalloproteinase-1 is regulated in tuberculosis by a p38 MAPK-dependent p-aminosalicylic acid-sensitive signaling cascade. J. Immunol. 182, 5865-5872 (2009).

38. Pargellis, C. et al. Inhibition of p38 MAP kinase by utilizing a novel allosteric binding site. Nat. Struct. Biol. 9, 268-272 (2002).

39. Marchenko, N. D. \& Moll, U. M. Mitochondrial death functions of p53. Mol. Cell. Oncol. 1, e955995 (2014).

40. Siegl, C. \& Rudel, T. Modulation of p53 during bacterial infections. Nat. Rev. Microbiol. 13, 741-748 (2015).

41. Mathupala, S. P., Heese, C. \& Pedersen, P. L. Glucose catabolism in cancer cells. The type II hexokinase promoter contains functionally active response elements for the tumor suppressor p53. J. Biol. Chem. 272, 22776-22780 (1997).

42. Gu, Z. T. et al. Heat stress induced apoptosis is triggered by transcriptionindependent $\mathrm{p} 53, \mathrm{Ca}(2+)$ dyshomeostasis and the subsequent $\mathrm{Bax}$ mitochondrial translocation. Sci. Rep. 5, 11497 (2015).

43. Zawacka-Pankau, J. et al. Inhibition of glycolytic enzymes mediated by pharmacologically activated p53: targeting Warburg effect to fight cancer. $J$. Biol. Chem. 286, 41600-41615 (2011).

44. Perfettini, J. L. et al. Essential role of p53 phosphorylation by p38 MAPK in apoptosis induction by the HIV-1 envelope. J. Exp. Med. 201, 279-289 (2005).

45. Sanchez-Prieto, R., Rojas, J. M., Taya, Y. \& Gutkind, J. S. A role for the p38 mitogen-acitvated protein kinase pathway in the transcriptional activation of p53 on genotoxic stress by chemotherapeutic agents. Cancer Res. 60, 2464-2472 (2000).

46. Wallis, R. S. Reconsidering adjuvant immunotherapy for tuberculosis. Clin. Infect. Dis. 41, 201-208 (2005).

47. Andree, M. et al. BID-dependent release of mitochondrial SMAC dampens XIAP-mediated immunity against Shigella. EMBO J. 33, 2171-2187 (2014).

48. Dickins, R. A. et al. Probing tumor phenotypes using stable and regulated synthetic microRNA precursors. Nat. Genet. 37, 1289-1295 (2005).

49. Matveeva, O. et al. Comparison of approaches for rational siRNA design leading to a new efficient and transparent method. Nucleic Acids Res. 35, e63 (2007).

\section{Acknowledgements}

We thank Prof. Thomas Langmann (Chair of Experimental Immunology of the Eye, University of Cologne) for providing BV-2 microglial cells. M. Chambon, D. Banfi, and N. Ballanfat (BSF, EPFL) were of great help during assay development and HTS. Prof. M. Fabri and Julia Steiger (Department for Dermatology, University Hospital of Cologne, Germany) assisted with human macrophage isolation. Pseudomonas strains and reagents were kindly provided by Prof. Arne Rietsch (Department of Molecular Biology and Microbiology, Case Western Reserve University, Cleveland, USA).This work was supported by the Thematic Translational Unit Tuberculosis (TTU TB, grant number TTU 02.806 and 02.905) of the German Center of Infection Research (DZIF). Financial support was received from the German Research Foundation (DFG RY 159) and the Center for Molecular Medicine Cologne (ZMMK-CAP8).

\section{Author contributions}

J.R. designed the study. J.G., E.vG., S.W., F.S., A.E., M.F., M.H., and M.S. performed biological experiments. I.S. provided patient samples. C.H., L.W., C.P., M.P., and H.K. generated knockout cell lines and mice. J.G. analyzed the data. J.G. and J.R. wrote the paper with input and approval from all authors.

\section{Additional information}

Supplementary Information accompanies this paper at https://doi.org/10.1038/s41467019-08405-9.

Competing interests: The authors declare no competing interests.

Reprints and permission information is available online at http://npg.nature.com/ reprintsandpermissions/

Journal peer review information: Nature Communications thanks Randall J. Basaraba and the other anonymous reviewer for their contribution to the peer review of this work.

Publisher's note: Springer Nature remains neutral with regard to jurisdictional claims in published maps and institutional affiliations.

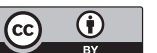

Open Access This article is licensed under a Creative Commons Attribution 4.0 International License, which permits use, sharing, adaptation, distribution and reproduction in any medium or format, as long as you give appropriate credit to the original author(s) and the source, provide a link to the Creative Commons license, and indicate if changes were made. The images or other third party material in this article are included in the article's Creative Commons license, unless indicated otherwise in a credit line to the material. If material is not included in the article's Creative Commons license and your intended use is not permitted by statutory regulation or exceeds the permitted use, you will need to obtain permission directly from the copyright holder. To view a copy of this license, visit http://creativecommons.org/ licenses/by/4.0/.

(C) The Author(s) 2019 